\title{
Integrated multi-omics analysis reveals common and distinct dysregulated pathways for genetic subtypes of Frontotemporal Dementia
}

\section{Kevin Menden}

German Center for Neurodegenerative Diseases

Margherita Francescatto

German Center for Neurodegenerative Diseases

Tenzin Niyma

German Center for Neurodegenerative Diseases

Cornelis Blauwendraat

National Institutes of Health https://orcid.org/0000-0001-9358-8111

\section{Ashutosh Dhingra}

German Center for Neurodegenerative Diseases

\section{Melissa Castillo-Lizardo}

German Center for Neurodegenerative Diseases

https://orcid.org/0000-0001-9350-8126

Noemia Fernandes

German Center for Neurodegenerative Diseases

\section{Lalit Kaurani}

German Center for Neurodegenerative Diseases

\section{Deborah Kronenbeerg-Versteeg}

University of Tübingen

\section{Burcu Atasu}

German Center for Neurodegenerative Diseases

\section{Eldem Sadikoglou}

German Center for Neurodegenerative Diseases

\section{Barbara Borroni}

University of Brescia

\section{Salvador Rodriguez-Nieto}

German Center for Neurodegenerative Diseases

Javier Simon-Sanchez

German Center for Neurodegenerative Diseases

\section{Andre Fischer}

German Center for Neurodegenerative Diseases

\section{David Craig}


Keck School of Medicine of University of Southern California

\section{Manuela Neuman}

German Center for Neurodegenerative Diseases

\section{Stefan Bonn}

University Medical Center Hamburg-Eppendorf https://orcid.org/0000-0003-4366-5662

\section{Patrizia Rizzu}

German Center of Neurodegenerative Diseases-Tübingen

\section{Peter Heutink ( $\sim$ peter.heutink@dzne.de )}

German Center for Neurodegenerative Diseases https://orcid.org/0000-0001-5218-1737

\section{Article}

Keywords: frontotemporal dementia (FTD), genetics

Posted Date: February 9th, 2021

DOl: https://doi.org/10.21203/rs.3.rs-153135/v1

License: (9) This work is licensed under a Creative Commons Attribution 4.0 International License. Read Full License 


\section{Integrated multi-omics analysis reveals}

2 common and distinct dysregulated

3 pathways for genetic subtypes of

${ }_{4}$ Frontotemporal Dementia

7 Kevin Menden ${ }^{1, *}(\mathrm{KM})$; Margherita Francescatto ${ }^{1}$ (MF); Tenzin Nyima ${ }^{1}$ (TN); Cornelis

8 Blauwendraat ${ }^{1,2}(\mathrm{CB})$; Ashutosh Dhingra ${ }^{1}(\mathrm{AD})$; Melissa Castillo-Lizardo ${ }^{1}(\mathrm{MC})$;

9 Noémia Fernandes ${ }^{1}$ (NF); Lalit Kaurani ${ }^{4}$ (LK); Deborah Kronenberg-Versteeg ${ }^{1,3}$

10 (DKV); Burcu Atarsu'1,3 (BA); Eldem Sadikoglou ${ }^{1}$ (ES); Barbara Borroni ${ }^{6}$ (BB);

11 Salvador Rodriguez-Nieto ${ }^{1}$ (SRN); Javier Simon-Sanchez ${ }^{1,3}$ (JSS); Andre Fischer ${ }^{4}$

12 (AF); David Wesley Craig ${ }^{7}$ (DWC); Manuela Neumann ${ }^{1}(\mathrm{MN})$; Stefan Bonn ${ }^{1,5}(\mathrm{SB})$;

13 Patrizia Rizzuำ (PR); Peter Heutink ${ }^{1, *}(\mathrm{PH})$.

\section{Affiliations}

1 German Center for Neurodegenerative Diseases, Tübingen, Germany 
5 Institute of Medical Systems Biology, University Medical Center Hamburg-Eppendorf,

21 Hamburg, Germany

226 Neurology Unit, Department of Clinical and Experimental Sciences, University of Brescia,

23 Italy

247 University of Southern California, California, USA

${ }^{*}$ corresponding authors

27 Peter Heutink: peter.heutink@dzne.de

28 Kevin Menden: kevin.menden@qbic.uni-tuebingen.de

30 Abstract

31 Understanding the molecular mechanisms underlying frontotemporal dementia (FTD) is essential for the development of successful therapies. Here we integrated transcriptomic and epigenomic analyses of postmortem human brains of FTD patients with mutations in MAPT,

34 GRN and C9orf72 and detected common and distinct dysregulated cellular pathways between patient groups. Our results highlight that excitatory neurons are the most vulnerable neuronal cell type and that vascular aberrations are a common hallmark in FTD. Via integration of multi-omics data, we detected several transcription factors and pathways which regulate the strong neuroinflammation observed in FTD-GRN. Small RNA-seq data and verification experiments in cellular models identified up-regulated miRNAs that inhibit cellular trafficking pathways in FTD and lead to microglial activation. These findings shed

41 light on novel mechanistic and pathophysiological hallmarks of FTD. The data represent the 
$42 \quad 1^{\text {st }}$ phase of a multi-omics, multi-model data resource for FTD research which allows in-

43 depth molecular research into disease mechanisms that will further mechanistic FTD

44 research.

\section{Introduction}

47 Frontotemporal Dementia (FTD) is a devastating pre-senile dementia characterized by progressive deterioration of the frontal and anterior temporal lobes ${ }^{1}$. The most common symptoms include severe changes in social and personal behaviour as well as a general blunting of emotions. Clinically, genetically, and pathologically there is considerable overlap with other neurodegenerative diseases including Amyotrophic Lateral Sclerosis (ALS), Progressive Supranuclear Palsy (PSP) and Cortical Basal Degeneration (CBD) ${ }^{2}$. Research into FTD has made major advances over the past decades. Up to $40 \%$ of cases ${ }^{3}$ have a positive family history and up to $60 \%$ of familial cases can be explained by mutations in the genes Microtubule Associated Protein Tau (MAPT), Granulin (GRN) and C9orf72 ${ }^{4}$ which has been key to the progress in our understanding of its molecular basis. Several other disease-causing genes have been identified that account for a much smaller fraction of cases ${ }^{5}$. Mutations in MAPT lead to accumulation of the Tau protein in neurofibrillary tangles in the brain of patients while mutations in GRN and C9orf72 lead to the accumulation of TDP $-43^{6}$, as well as dipeptide repeat proteins (DPRs) and RNA foci in the case of C9orf72 ${ }^{7}$.

61 As of today, no therapy exists that halts or slows the neurodegenerative process of FTD and in order to develop successful therapies there is an urgent need to determine whether a common target and therapy can be identified that can be exploited for all patients, or

64 whether the distinct genetic, clinical and pathological subgroups need tailored treatments.

65 Therefore, the development of remedies relies heavily on a better understanding of the molecular and cellular pathways that drive FTD pathogenesis in all FTD subtypes. 
67 Although our knowledge of FTD pathogenesis using molecular and cellular biology approaches has significantly advanced during recent years, a deep mechanistic understanding of the pathological pathways requires simultaneous profiling of multiple regulatory mechanisms. As neurodegenerative diseases develop over time, it is furthermore important to examine temporal changes. While post-mortem human brain tissue represents the end-stage of disease, well-defined rodent models can be used to address the temporal component. Lastly, experimental validation of derived hypotheses can be achieved in cellular systems, such as neurons derived from induced pluripotent stem cells (iPSCs) as well as in rodent models.

The Risk and modifying factors in Frontotemporal Dementia (RiMod-FTD) consortium ${ }^{8}$ generates a multi-model and multi-omics data resource with the focus on mutations in the three most common causal genes: MAPT, GRN and C9orf72. The data resource will consist of multi-omics datasets from multiple post-mortem human brain regions, and matching iPSC derived neurons and brain tissue of transgenic mouse models at different time points.

81 Here, we report on data derived from the post-mortem human brain RNA-seq, CAGE-seq, smRNA-seq and methylation datasets from RiMod-FTD. We identified dysregulation of overlapping pathways in all disease groups that indicates converging disease mechanisms manifesting during disease progression. Using deconvolution analysis, we have identified changes in cellular composition that are either common or distinct to genetic subgroups.

86 Through integration of smRNA-seq and CAGE-seq data, we could furthermore highlight 87 potential regulatory molecules that might play important roles in FTD pathogenesis, within the identified functional gene modules and pathways. 


\section{Results}

\section{Multi-omics Data Resource for Frontotemporal Dementia}

91 We have analysed data from brain tissue from diseased patients carrying mutations in the

92 MAPT $(n=17)$, GRN ( $n=11)$ or C9orf72 $(n=17)$ genes and non-demented controls $(n=16)$.

93 The average age of FTD groups was lower than that of healthy controls (Table S1). We

94 obtained tissue from up to 7 regions for each brain. The temporal and frontal lobes are the

95 most affected areas in FTD, but we also obtained material from the occipital lobe,

96 hippocampus, caudate, putamen and cerebellum for verification experiments. We performed

97 CAGE-seq, smRNA-seq and quantitative proteomics (Miedema et al., manuscript in preparation) on tissue from frontal and temporal lobes, and generated methylation and RNA-

99 seq data for frontal lobe tissue. Additional data types such as ATAC-seq, transgenic mouse

100 models and iPS derived data are planned for future releases. The resulting, comprehensive

101 multi-omics data resource enables the study of disease mechanisms in FTD subtypes to

102 greater detail than single genomics experiments (Fig. 1). 
a Multi-model Sample Preparation
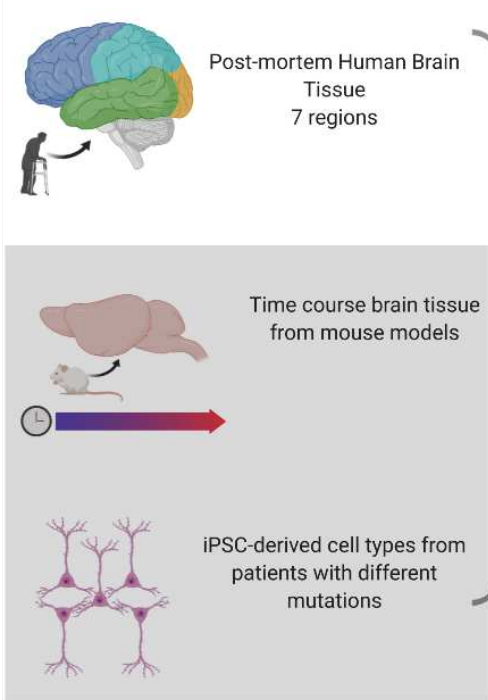

b

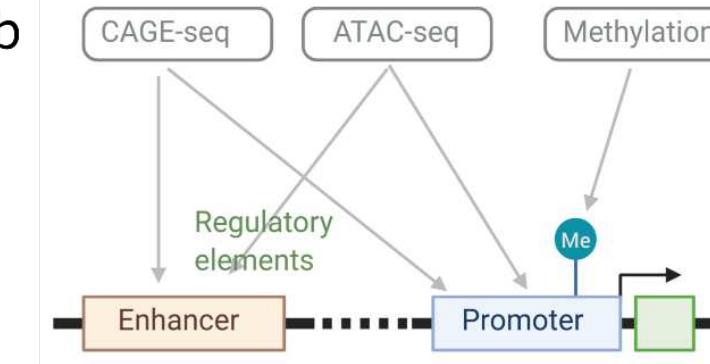

Multi-omics Profiling
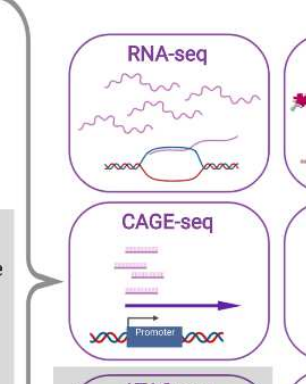

ATAC-seq

$\ldots$

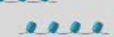

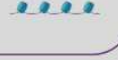

1

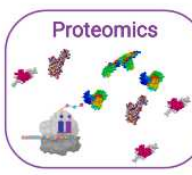

smRNA-seq

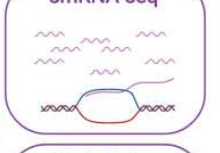

Methylation

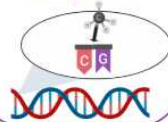

Gene expression
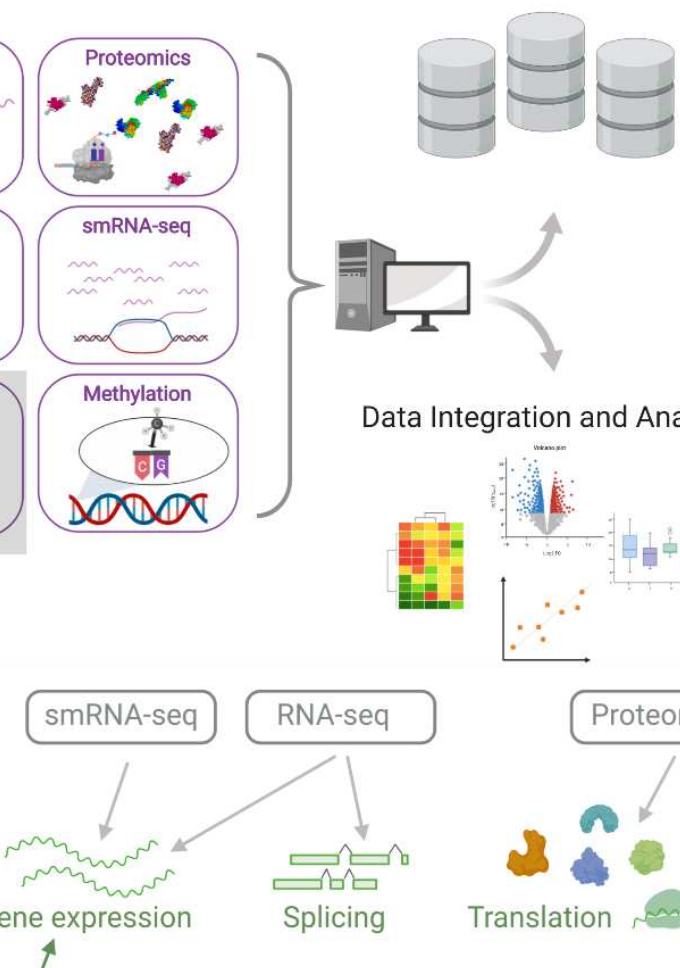

Data Integration and Analysis Gene
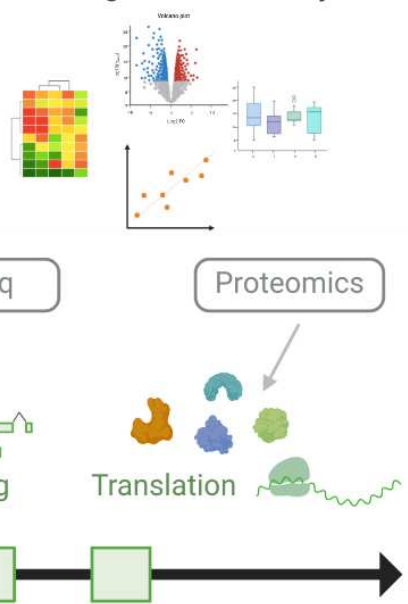

Figure 1: Graphical overview of the RiMod-FTD project. a In phase 1, Human Postmortem brain tissue samples from multiple regions of patients with mutations in GRN, MAPT and C9orf72 have been collected and used for multi-omics data generation. Grey shading indicates datasets that will be completed in future phases. The datasets have been integrated and analysed and published to be accessible as FTD resource. In subsequent phases additional datatypes will be added (i.e. ATAC-seq) and the resource will be extended with data from matching mouse models and iPSC derived celltypes. $\mathbf{b}$ The multi-omics approach allows to profile multiple regulatory features of gene expression, including enhancer- and promoter-based regulation, epigenetic regulation, alternative splicing, posttranscriptional regulation (miRNAs) and regulation of translation (proteomics). 
116 In the current study, we have integrated RNA-seq, CAGE-seq, smRNA-seq and methylation

117 data from the Gyrus Frontalis Medialis (GFM) (Table S2), as this brain region is strongly 118 affected in FTD.

\section{Differential gene expression analysis and cellular deconvolution}

\section{1 of the GFM in FTD.}

122 To identify general gene expression patterns in the GFM of patients with FTD, we performed differential gene expression (DGE) and principal component analysis (PCA) using RNA-seq data. The PCA indicates considerable heterogeneity between samples, as can be expected from post-mortem human brain tissue (Fig. 2a). However, a difference between FTD cases and control samples is clearly visible. Differentially expressed genes (DEGs) were calculated for all disease groups (FTD-MAPT, FTD-GRN, FTD-C9orf72) compared to controls while controlling for gender and $\mathrm{pH}$-value (see Methods). We observed the largest number of DEGs (adj. P-value < 0.05) for FTD-GRN, followed by FTD-MAPT and FTD-C9orf72 (Fig. 2b). DGE of smRNA-seq data yielded 78, 21 and 39 differentially expressed miRNAs in FTD-MAPT, FTD-GRN and FTD-C9orf72, respectively (Fig. 2c).

133 Due to the neurodegenerative nature of FTD, it is likely that there exists a systematic 134 difference in cell composition between cases and controls which can affect DGE analysis due to differences in gene expression between cell types - a problem which has often been overlooked in tissue expression studies. Here, we account for this problem by applying a conservative filtering approach and removing DEGs that are associated with changing cellular composition (see Methods). All further analyses were based on the filtered set of DEGs, unless otherwise specified. Note that this method could only be applied to the total 
140 RNA-seq dataset because similar cell type specificity data (here, single-cell RNA-seq data)

141 was not available for other data types.

143 Activation of extracellular matrix (ECM) associated pathways

144 and circulatory system development.

145 We next performed pathway enrichment analysis with DEGs from the RNA-seq data using

146 go:Profiler ${ }^{9}$ to identify the most affected cellular pathways. Down-regulated genes are

147 strongly enriched for mitochondrial and oxidative phosphorylation pathways in both FTD-

148 GRN and FTD-MAPT (Fig. 2d, Fig. S1), indicating a dysfunctional energy metabolism - a

149 well-known hallmark of many neurodegenerative diseases ${ }^{10}$. Neuronal system pathways are

150 enriched among down-regulated genes for both groups as well. This might be explained by

151 dysfunctional neurons that have not yet undergone apoptosis or by a general impairment of

152 neuronal function caused by the disease. Other significantly down-regulated pathways

153 include ubiquitin-dependent protein metabolism and vesicle-mediated transport (FTD-GRN).

154 In all three groups, up-regulated genes are enriched for extracellular matrix (ECM)

155 associated pathways and circulatory system development (Fig. S1). Genes involved in

156 Hippo-signalling are enriched in FTD-GRN and FTD-MAPT (Fig. 2d), and immune system

157 related genes are enriched in FTD-GRN. ECM dysregulation, in particular, has been

158 implicated with several neurodegenerative diseases. For instance, studies in mouse models

159 showed that tau pathology can lead to ECM reorganization and that reducing ECM proteins

160 could reverse memory deficits in an AD mode ${ }^{11,12}$. While the role of the ECM in FTD

161 remains unknown, our results suggest a prominent involvement in end-stage FTD.

We also specifically examined the DEGs with the largest fold-changes in the RNA-seq data because large expression fold-changes often signify strong dysregulation. These results 
support the importance of ECM in FTD as for all disease groups, multiple matrix

166 metalloproteinase enzymes (MMPs) are among the DEGs with the largest LFCs (Fig. S2,

167 Fig. S3A). Elevated RNA levels of MMP genes have been reported for many

168 neurodegenerative diseases, and MMPs target a wide range of ECM ${ }^{13}$ indicating their

169 importance in neurodegenerative mechanisms ${ }^{14}$. Protein interaction networks of up-

170 regulated genes in FTD-MAPT and FTD-GRN show the central importance of MMPs in

171 these networks (Fig. S3 B \& C).

172

173 


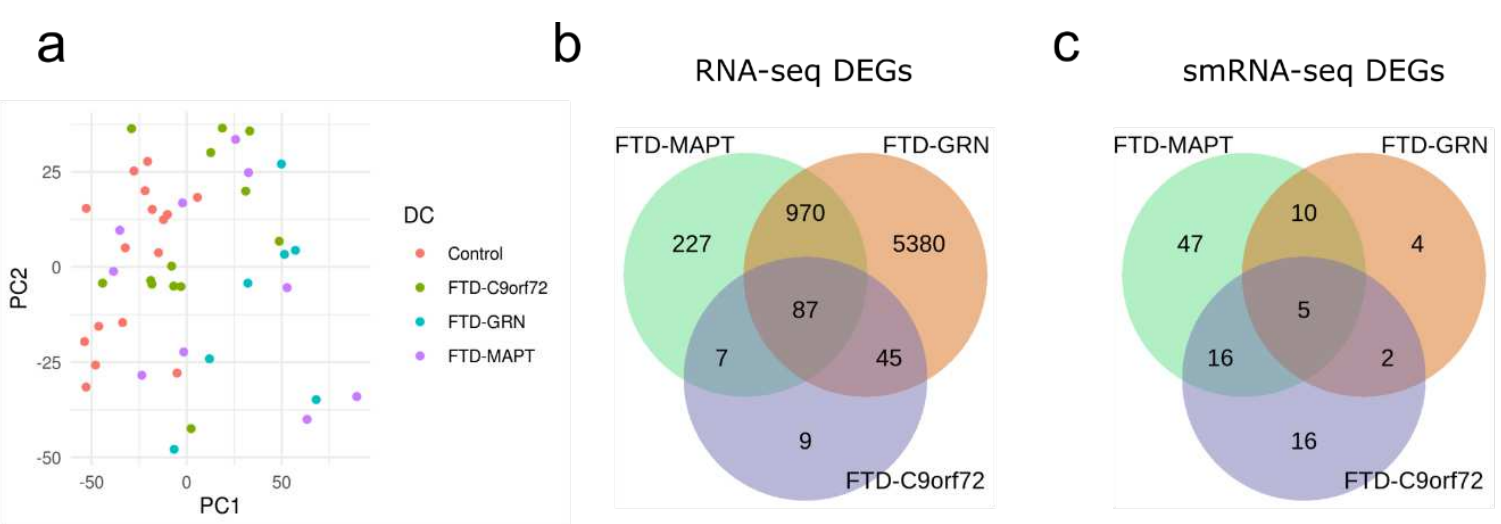

d

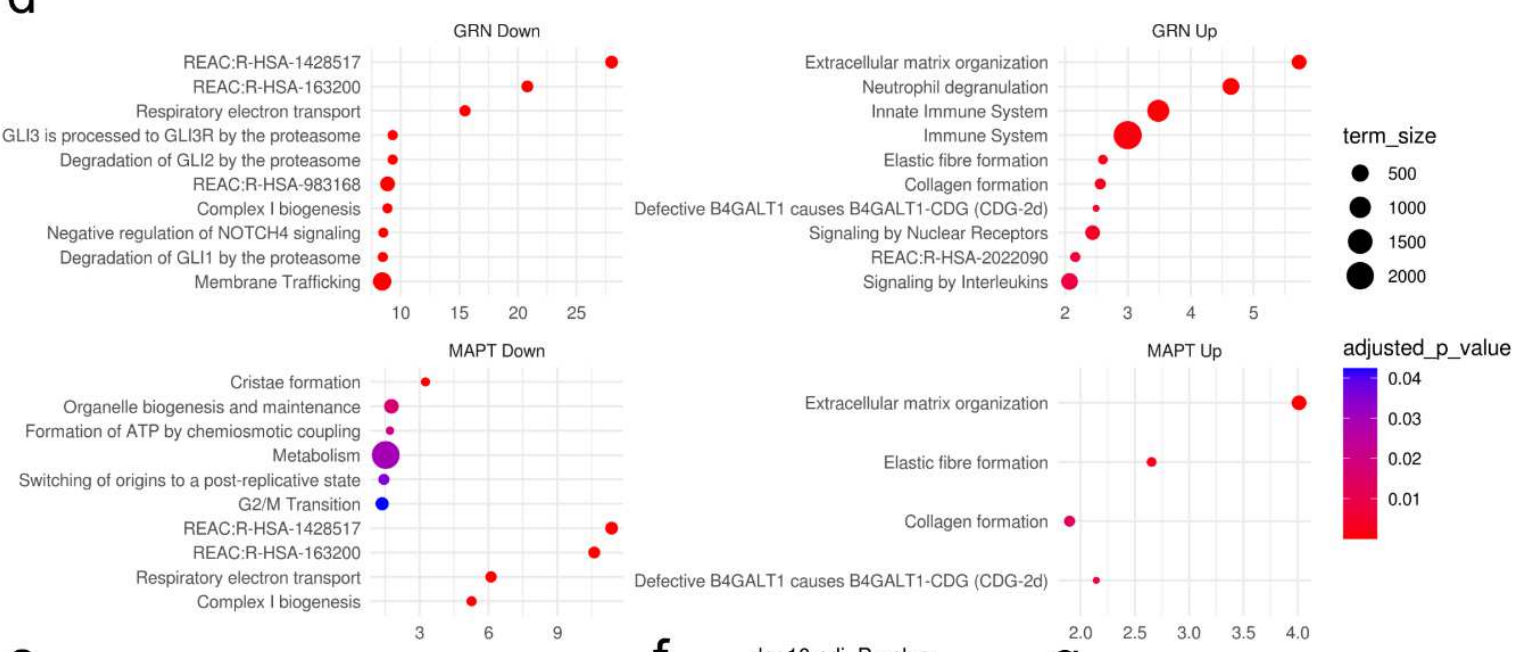

e

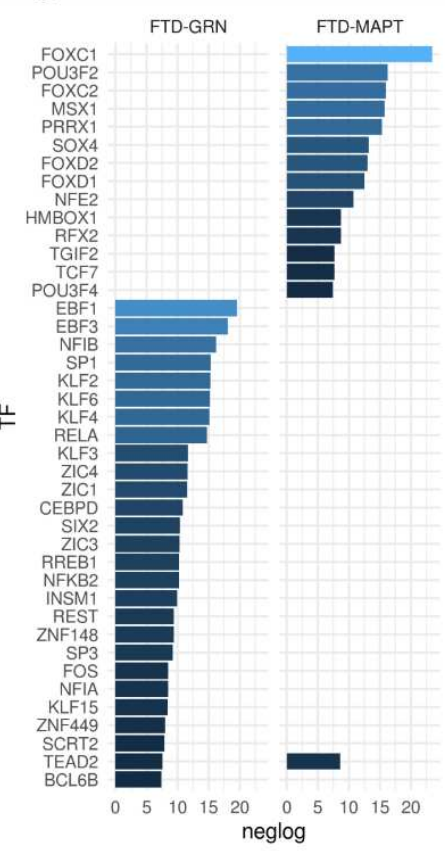

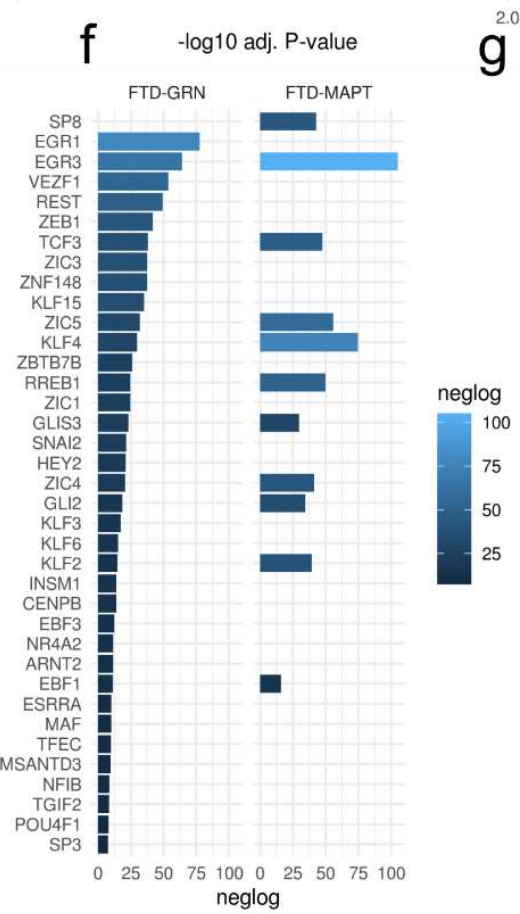

9

DMP Overlap

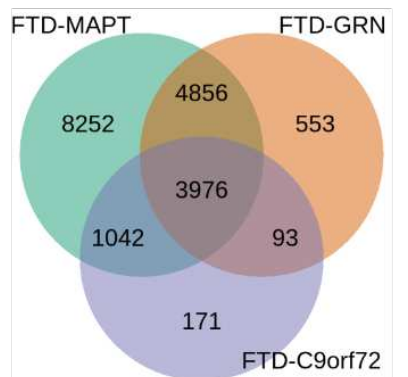

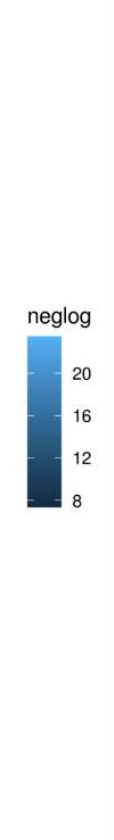

175 Figure 2: Gene- and Pathway-level transcriptional changes in FTD. a Principal component analysis of variance stabilized RNA-seq expression values, coloured by group. $\mathbf{b}$ 
177 Overlap between RNA-seq DEGs from different disease groups. c Overlap between

178 smRNA-seq DEGs from different disease groups. d Enriched Reactome pathways in RNA-

179 seq up- and down-regulated DEGs. Shown are the ten most significant pathways per group;

180 the $\mathrm{x}$-axis signifies the negative log10 P-value. Colour corresponds to adjusted P-value and

181 node size corresponds to the number of genes in a pathway. e,f Best candidates for active

182 and inactive TFs in FTD-GRN and FTD-MAPT, respectively. The x-axis signifies the

183 negative log10 P-value. g Overlap of DMPs in different disease groups.

\section{Regulatory mechanisms associated with differential expression}

186 To better understand relevant regulatory mechanisms leading to these gene expression 187 changes, we generated a set of candidate driver transcription factors (TFs) using the GFM

188 CAGE-seq data. CAGE-seq cluster counts, when assigned to the closest gene, correlate 189 well with RNA-seq expression data (average sample-wise correlation coefficient: 0.6, Fig.

190 S4). We used the CAGE-seq data to predict candidate driver TFs for up- and downregulated genes (see Methods for details). TEAD2, a TF central to the Hippo signalling pathway, is the only predicted active TF common to FTD-GRN and FTD-MAPT (Fig. 2e), while there is greater overlap among inactive TFs (here: inactive TF = has down-regulated targets, Fig. 2f). Moreover, we performed miRNA-target gene mapping to evaluate potential regulatory roles of miRNAs. Expression values of miRNAs were correlated with their predicted targets using matching samples from the RNA-seq data. Only miRNA-target pairs with considerable negative correlation were retained (see Methods).

DNA methylation is another important regulatory mechanism that can affect gene expression. We generated Illumina Infinium EPIC methylation data from the GFM and considered the most variable CpG sites $(28,173)$ corrected for possible confounding effects 
using surrogate variable analysis (SVA) to perform differential methylation analysis (see Methods). We detected $18,126,9,478$ and 5,282 significantly differentially methylated positions (DMPs) for FTD-MAPT, FTD-GRN, and FTD-C9orf72, respectively (Fig. 2g). The C9orf72 repeat expansion is known to be associated with hypermethylation ${ }^{15}$ and we confirmed in our data that a CpG site located at the 5'-end of the C9orf72 gene, only $14 \mathrm{bp}$ away from the repeat expansion, is hypermethylated (log fold-change: 0.6, Fig. S4A). Pathway enrichment analysis of genes in proximity to DMPs yielded enrichment of genes involved in nervous system development for hypermethylated CpG sites. Genes close to hypomethylated sites were enriched for system development and vasculature development (Fig. S5C). As hypermethylation of CpG sites at promoter regions is associated with decreased expression, this indicates epigenetically controlled expression inhibition of genes important for neuronal function, or remnants of cell composition effects that could not be entirely alleviated by SVA (see Methods). Performing biological age prediction using the methylation data resulted in underestimated age predictions for all groups, albeit to a lesser extent for FTD groups, which indicates accelerated aging in FTD (Fig. S5B).

\section{Vulnerability of excitatory neurons and enrichment of}

\section{endothelial cells}

To identify vulnerable cell types and disease-related cell composition changes, we inspected the results from the RNA-seq deconvolution analysis (Methods) with respect to genetic FTD subtypes. As expected, fractions of neuronal cells are systematically lower in all FTD groups compared to controls (Fig. S6). Consequently, virtually all other cell types show increasing percentages. We therefore calculated the percentage-wise change for each cell type and assessed statistical significance (see Methods). Strongest neuronal loss was observed in FTD-GRN, followed by FTD-MAPT and FTD-C9orf72 (Fig. 3a, Table S3), which agrees with studies that have shown that the frontal lobe is most strongly affected in FTD-GRN ${ }^{16-18}$. Moreover, neuronal loss can be primarily attributed to loss of excitatory neurons, while 
fractions of inhibitory are not significantly different to controls (Table S3). Our results confirm

229 findings from recent studies that found excitatory neurons to be especially vulnerable to tau

230 pathology ${ }^{19}$ and we specifically detected an important role of glutamatergic

231 neurotransmission in FTD ${ }^{20,21}$. Closer examination of the KEGG pathway 'glutamatergic synapse' suggests that AMPA receptors are mainly affected, while we could not see signs of dysregulation for NMDA receptors (Fig. S7 A-C). Analysis of candidate regulator TFs highlighted the TF Early Growth Response 3 (EGR3), targets of which are enriched for glutamatergic synapse genes (Fig. S7D), indicating involvement in excitatory neuronal function.

To validate our computational deconvolution, we considered the fractions of excitatory neurons as a proxy of neurodegeneration and correlated them with manually determined degeneration scores by a neuropathologist (Fig. 3b, Methods). Indeed, excitatory neuron fractions show strong negative correlation with pathology scores (Pearson's correlation coefficient $=-0.78, \mathrm{P}$-value $=2.8 \mathrm{e}-07$ ), thereby providing experimental confirmation of our computational predictions.

The strongest growth in percentage compared to the baseline is observed for endothelial cells in FTD-MAPT and FTD-C9orf72 disease groups, but not FTD-GRN, where microglial cells show the strongest increase. Circulatory system development is among the most significantly up-regulated biological processes in all three disease groups (Fig. 3 c \& d). The role of the circulatory system in FTD is relatively unexplored. However, Bennet et al. recently found increased vasculature growth in mouse models of FTD-MAPT with a P301L mutation $^{22}$. Interestingly, endothelial enrichment in FTD-MAPT is particularly strong in patients with a P301L mutation (Fig. 3a). Another recent study observed a particular microvascular structure with increased frequency in brains of patients with frontotemporal 
a

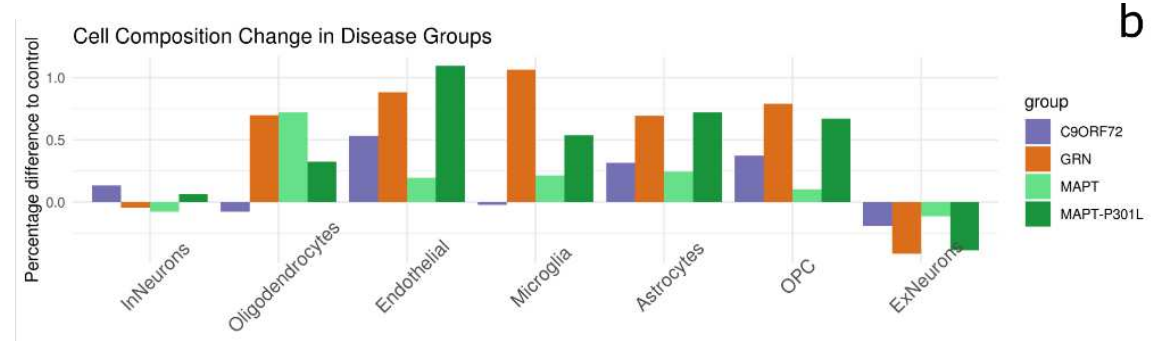

C

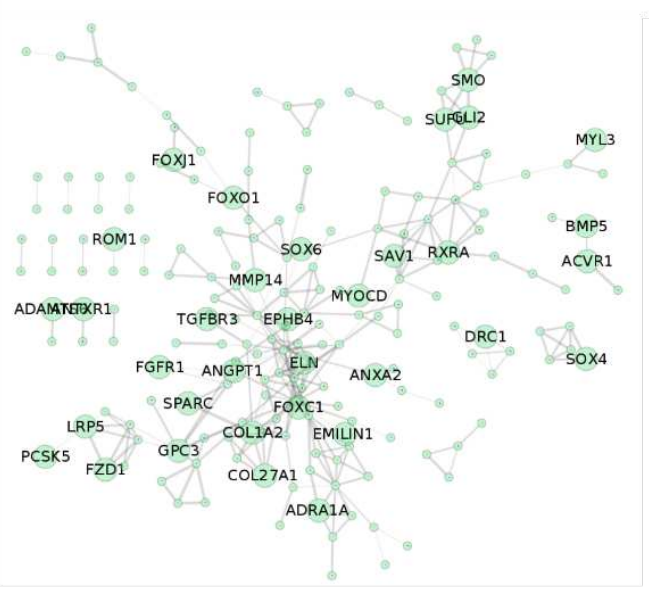

b

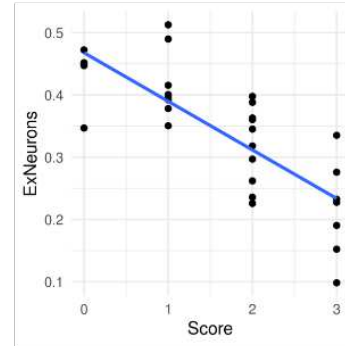

d

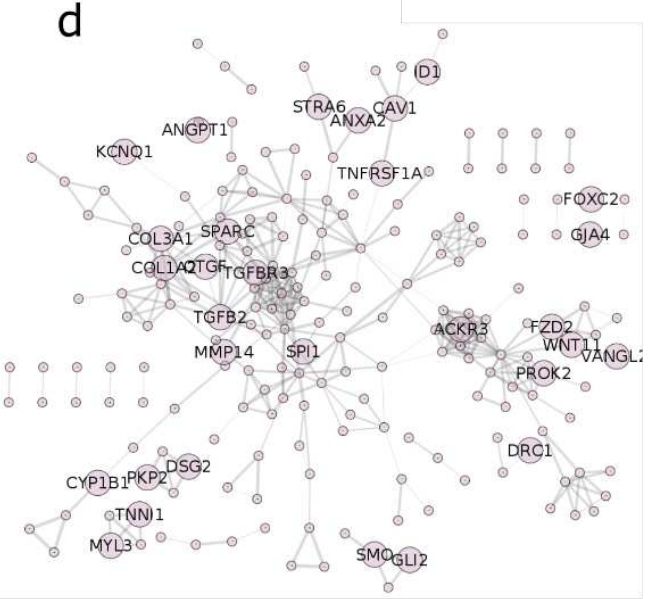

e
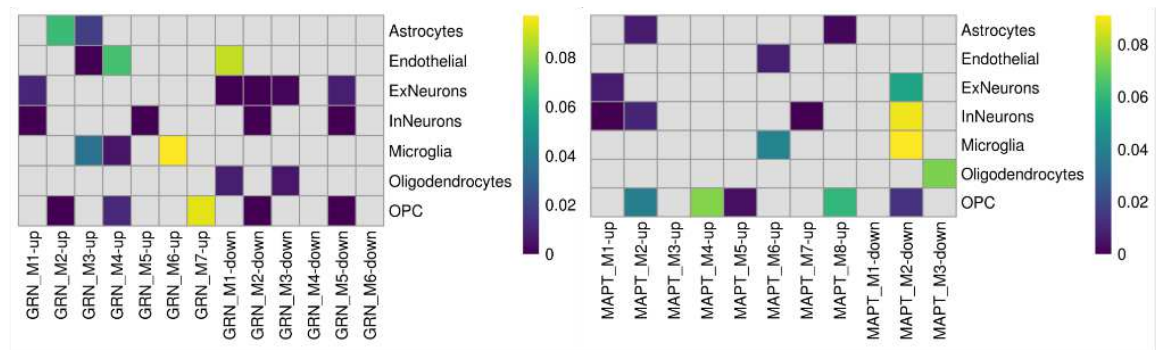

259 Figure 3: Cell composition changes in FTD. a Percentage change of averaged fractions

260 per group compared to the average of the control group. Genetic subtypes are indicated with different colours. b Regression of excitatory neuron fractions (y-axis) against neuropathology scores (x-axis). c, d PPI networks of genes up-regulated in FTD-MAPT and FTD-GRN (logfold-change > 1), respectively. Genes involved in the biological process "circulatory system development" are labelled. e Heatmap of EWCE analysis results for HumanBase modules of 
FTD-GRN and FTD-MAPT. Different modules are lined up on the x-axis, different cell types on the y-axis. Tile colour signifies the EWCE P-value. Tiles with P-values above 0.1 are marked grey.

To better understand transcriptional changes and regulatory mechanisms, we performed tissue-specific functional module detection with HumanBase ${ }^{25}$ and assessed cell type specificity of modules using EWCE ${ }^{26}$. Both for FTD-MAPT and FTD-GRN, most modules show specificity for a few cell types (Fig. 3e). Up-regulated modules in both groups are significantly enriched for endothelial genes $(P$-value $<0.1)$. Genes within these modules have been associated with blood vessel development (FTD-MAPT M6-up) and endothelial cell growth (FTD-GRN M4-up) by HumanBase (Fig. 3 c \& d), further supporting a distinct involvement of endothelial genes in these FTD subtypes.

\section{Increased Inflammatory response in FTD-GRN}

In patients with FTD-GRN, microglial fractions show an even larger relative increase than endothelial cells, indicating increased microglial activity. The protein encoded by GRN is well known for its importance to lysosomal function, is highly expressed in microglia and wellknown for having important functions in the immune system ${ }^{27,28}$. Microglia are also slightly enriched in FTD-MAPT $(P$-value $=0.037)$ but not in FTD-C9orf72 $(P$-value $=0.475)$.

Here, we have observed a prominent increase in microglial cell fractions and up-regulation of immune system pathways in FTD-GRN, a feature of GRN deficiency that has been frequently shown in mouse models ${ }^{28-30}$. We therefore wanted to further characterize potential underlying regulatory mechanisms. First, we examined FTD-GRN modules for enrichment of immune system-related terms. Indeed, several up-regulated modules are 
290 enriched for genes related to the immune system, while we could not find enrichment among

291 down-regulated modules. The module FTD-GRN M1-up contains genes important for

292 neutrophil migration and response to interleukins (Fig. 4 a \& d). Both modules M3-up and

293 M4-up contain genes relevant to NF-kappa-B (NFkB) signalling, as well as genes involved in

294 tumour necrosis factor (TNF) production (Fig. 4 b \& c, respectively). Finally, the module M6-

295 up is enriched for genes involved in T cell activation. Modules M3-up, M4-up and M6-up are

296 furthermore enriched for microglial-specific genes (Fig. 3e). Interestingly, several

297 necroptosis-related genes are up-regulated (M1-up: TLR3, TLR8, RIPK3; M4-up: RIPK2),

298 suggesting this pathway as a potential driver of neuronal death. While we did not detect

299 prominent signals for neuroinflammation in FTD-MAPT, the FTD-MAPT module M3-up

300 contains several genes involved in T cell and TNF signalling (EZR, RAB29, CARD8, HIPK1).

301 However, neuroinflammation is much less prominent in FTD-MAPT and FTD-C9orf72

302 compared to FTD-GRN.

303

304 


\section{a}
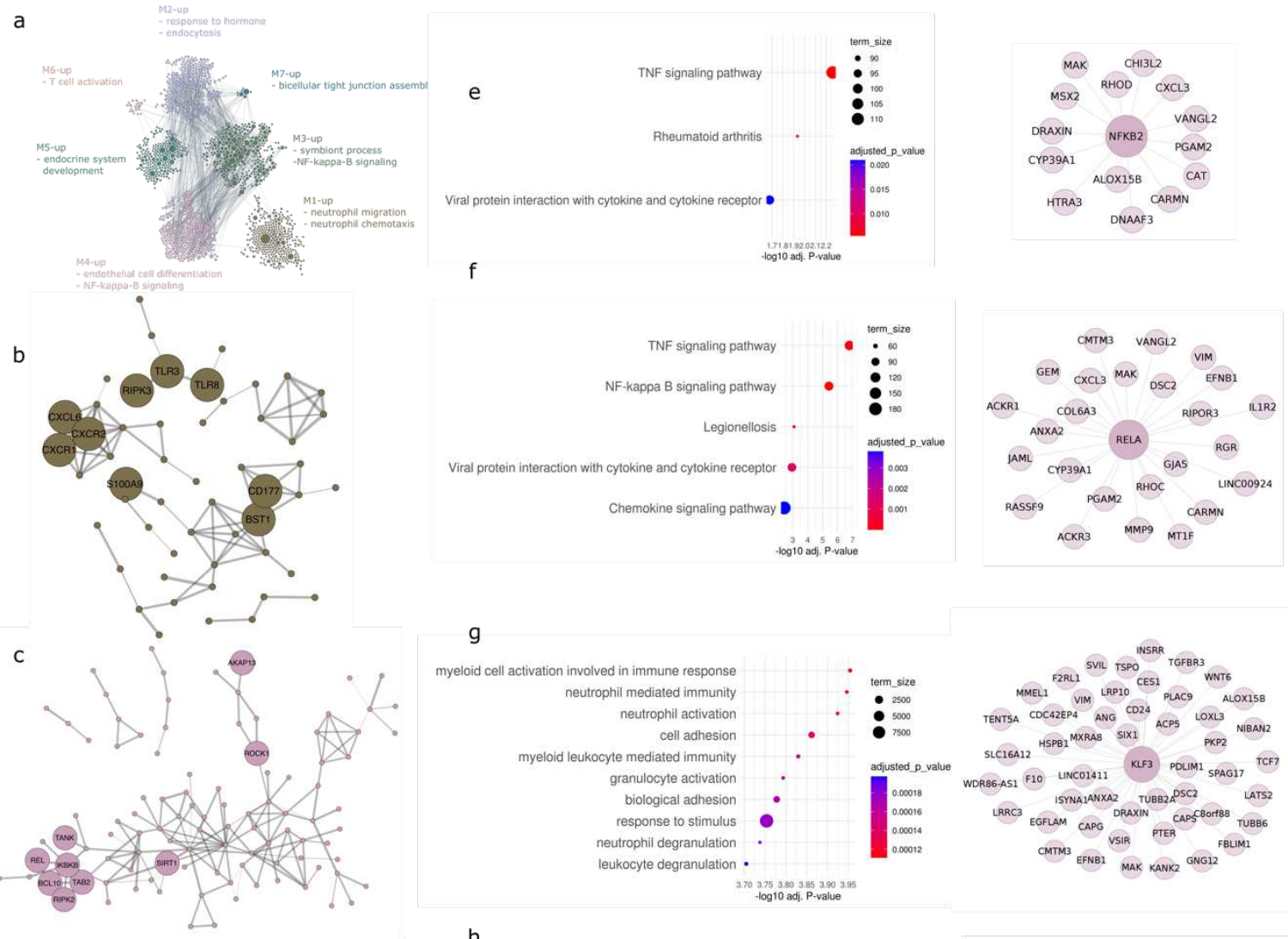

g

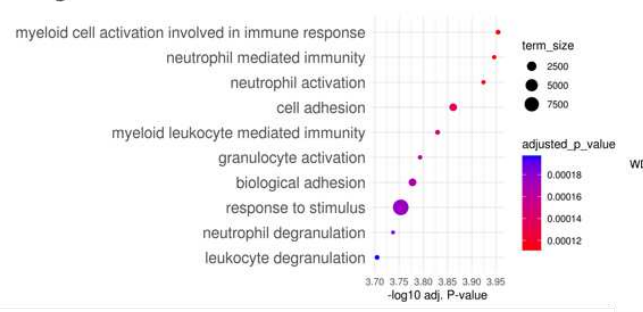

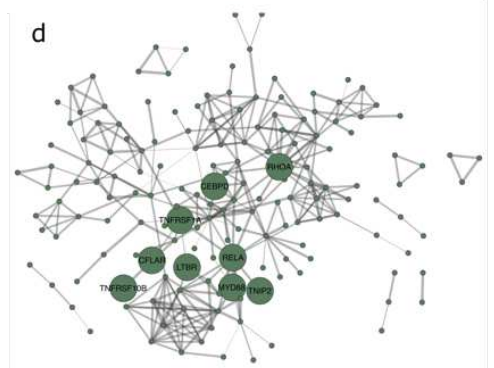

h

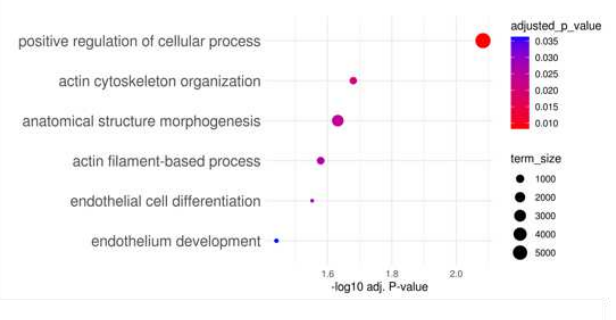

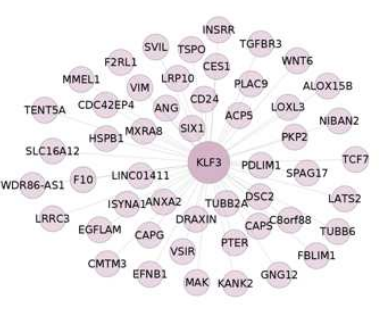

-

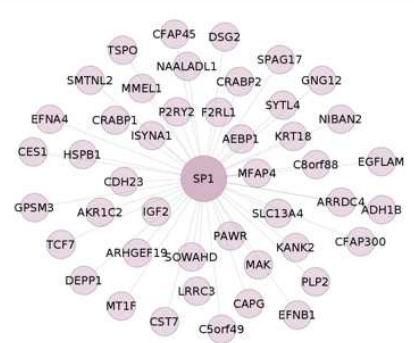

Figure 4: Neuroinflammation in FTD-GRN. a Up-regulated HumanBase modules in FTD-

307 GRN with most significant terms. b Protein-protein interaction (PPI) network (made with

308 String-DB) of FTD-GRN M1 up-module. Genes involved in necroptosis, interleukin response 309 and neutrophil migration are indicated c PPI network of FTD-GRN M4-up module. Genes

310 involved in NFkB signalling are indicated. d PPI network of FTD-GRN M3-up module. Genes

311 involved in NFkB signalling and CEPBD are indicated. $\mathbf{e}$ and $\mathbf{f}$ KEGG pathway enrichment of

312 predicted targets of TFs NFKB2 and RELA, respectively. $\mathbf{g}$ and $\mathbf{h}$ GO:BP pathway

313 enrichment of predicted targets of TFs KLF3 and SP1, respectively. 
316 Inspection of our candidate regulator TFs indicated the TFs Nuclear Factor Kappa B Subunit

3172 (NFKB2) and RELA which together form the NFkB signalling complex, as potential drivers

318 in FTD-GRN (Fig. 2e). Enrichment analysis of predicted NFKB2 and RELA targets in FTD-

319 GRN indeed revealed TNF signalling and NFkB signalling as the most significantly enriched

320 KEGG pathways (Fig. 4 e \& f). Furthermore, enrichment analysis indicated targets of the TFs

321 SP1 and KLF3 as highly enriched among genes in the FTD-GRN M3-up module. Predicted

322 KLF3 targets are enriched for immune system genes (Fig. 4g). SP1 target genes do not

323 show a strong enrichment but have roles in actin cytoskeleton organization and endothelial

324 cell differentiation, among others (Fig. 4h). We also investigated predicted targets of down-

325 regulated miRNAs and genes proximal to hypomethylated $\mathrm{CpG}$ sites for involvement in the

326 immune system in FTD-GRN but could not detect any significant immune system-relevant

327 enrichment suggesting they do not play a major role in regulating the immune response.

To closer examine which parts of the NFkB and TNF signalling pathways are affected in FTD-GRN and in FTD in general, we inspected fold-changes of genes from the corresponding KEGG pathways. Interestingly, the pro-inflammatory cytokine Interleukin 1 Beta (IL1B) is down-regulated in all disease groups, although only significantly in FTD-MAPT (Fig. S9A). Similarly, the inflammatory cytokine Interleukin 6 (IL6) has negative fold-changes in all disease groups. Downstream effector genes with positive fold-changes include multiple chemokines, Interleukin 18 Receptor 1 (IL18R1) and several metalloproteinases. leads to NFkB overactivation in microglia in a GRN loss-of-function mouse model ${ }^{29}$. 
340 behaviour (OCB) and inhibition of NFkB was sufficient to rescue the behavioural symptoms

341 linking these TFs to the OCB observed in patients.

\section{Dysfunctional energy metabolism and cellular trafficking in FTD}

343 Among the most significantly down-regulated pathways in FTD-GRN and FTD-MAPT are

344 several pathways involved in energy metabolism and oxidative phosphorylation (Fig. 2d, Fig.

345 S1). Inspection revealed the modules FTD-GRN M1-down and FTD-MAPT M1-down as

346 being most significantly associated with the term NADH dehydrogenase complex assembly

347 (Fig. 5 d \& e, Tables S4 and S5). Further inspection of the FTD-MAPT and FTD-GRN M1-up

348 modules revealed that they contain several NADH:Ubiquinone Oxidoreductase Subunit

349 genes (Fig. 5 b \& c), which are necessary for functional oxidative phosphorylation and hence

350 energy production. The FTD-GRN module is moreover enriched for genes involved in

351 intracellular transport and autophagy. The FTD-GRN M1-down module contains several

352 genes associated with FTD or ALS: Superoxide Dismutase 1 (SOD1), Dynactin Subunit 1

353 (DCTN1), PTEN Induced Kinase 1 (PINK1), Huntingtin (HTT), and CHCHD10. All these

354 genes show lower expression values in every genetic subgroup, although they do not reach

355 significant levels in all groups (Fig. 5a). 


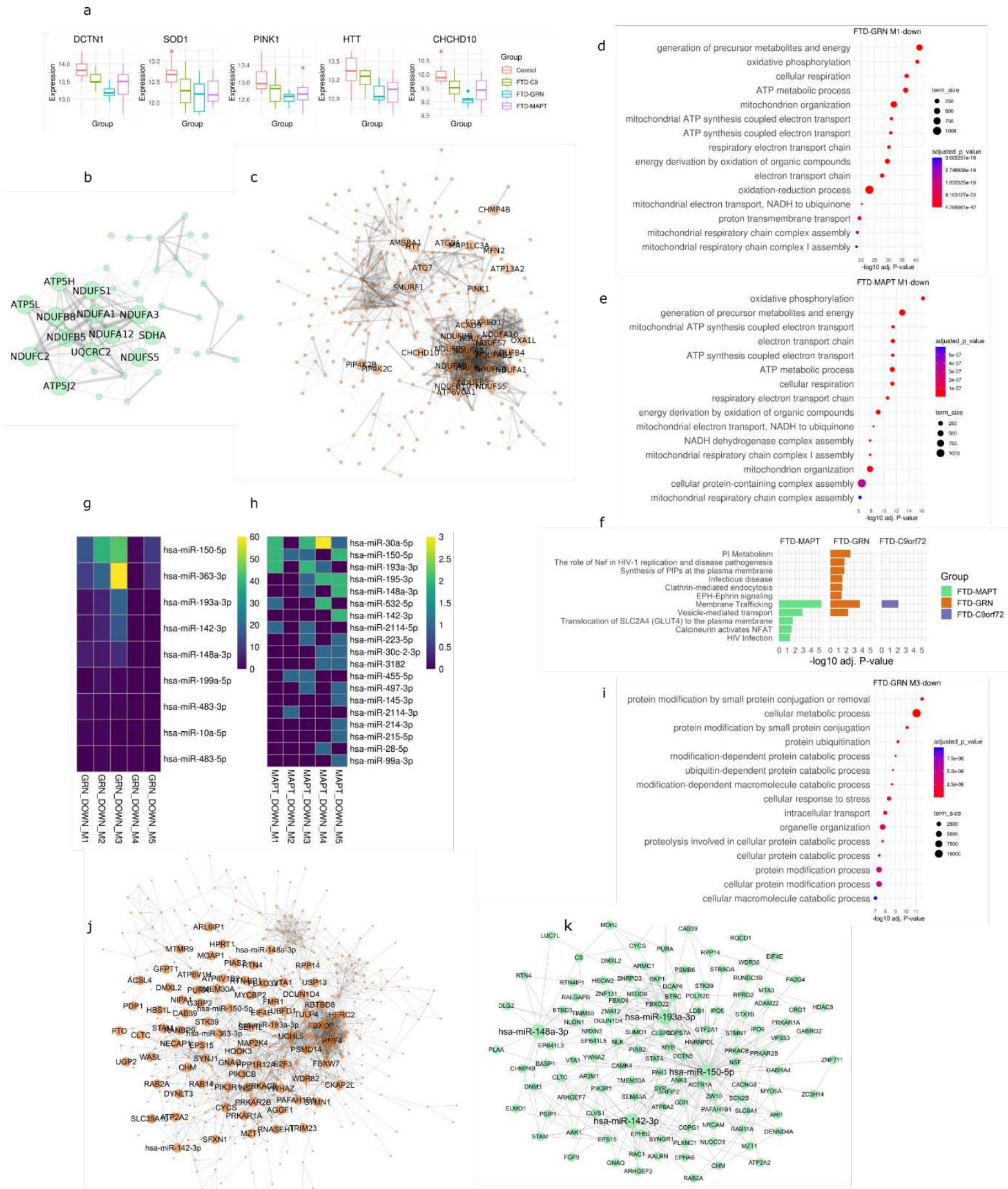

358 Figure 5: Impaired oxidative phosphorylation and cellular trafficking in FTD. a

359 Expression levels (variance stabilized with DESeq2) of the genes CHCHD10, PINK1, SOD1,

360 DCTN1 and HTT in different groups. b STRING-DB PPI of FTD-MAPT M1-down module.

361 Genes involved in oxidative phosphorylation are labelled. c PPI of FTD-GRN M1-down 
module. Genes involved in NADH dehydrogenase complex assembly and mitophagy are

363 labelled, as well as CHCHD10. d, e Most significant results from pathway enrichment analysis with g:Profiler (GO biological process) for the FTD-GRN M1-down module and the FTD-MAPT M1-down module, respectively. Node colour corresponds to adjusted P-value and node size to term size. $\mathbf{f}$ Most significant results from enrichment analysis (Reactome) of targets of up-regulated miRNAs in all disease groups. $\mathbf{g}, \mathbf{h}$ Heatmaps of intersection-overunion scores between predicted miRNA targets and down-regulated modules in FTD-GRN and FTD-MAPT, respectively. i Top enrichment results of g:Profiler (GO biological process) for the FTD-GRN M3-down module. Node colour corresponds to adjusted P-value and node size to term size. j PPI network (String-DB) of FTD-GRN M3-down module. Predicted targets of up-regulated miRNAs are labelled. $\mathbf{k}$ PPI network of predicted targets of up-regulated miRNAs in FTD-MAPT.

Cellular transport is thought to play a key role in FTD pathogenesis as impaired trafficking can affect protein and mitochondria homeostasis. Here, we show that mitochondrial function is strongly impaired in end-stage FTD and that transport pathways are tightly connected to this pathology. We thus looked for potential regulatory mechanisms driving the pathological changes. Enrichment analysis of targets of up-regulated miRNAs in all disease groups revealed cellular localization as the most significantly enriched biological process (GO:BP) and membrane trafficking as the most significant Reactome pathway (Fig. 5f). Up-regulated miRNAs in FTD therefore seem to primarily target cellular transport pathways and might play important roles in dysfunctional transportation.

To detect modules and genes predominantly targeted by up-regulated miRNAs, we calculated the intersection-over-union (IoU) of up-regulated miRNA targets with downregulated modules for FTD-GRN and FTD-MAPT (Fig. $5 \mathrm{~g} \& \mathrm{~h}$ ). The FTD-GRN M3-down 
module is most strongly targeted by miRNAs and contains genes involved in metabolic processes and cellular localization (Fig. 5i, Table S4). Five miRNAs have putative target genes in this module: hsa-miR-150-5p, hsa-miR-142-3p, hsa-miR-193a-3p, hsa-miR-148a$3 p$ and hsa-miR-363-3p, which are all significantly up-regulated in FTD-MAPT as well, except hsa-miR-363-3p. We generated networks of the above-mentioned candidate miRNAs combined with a PPI network of the FTD-GRN M3-down module (Fig. 5j) and a PPI network of all predicted targets in FTD-MAPT (Fig. 5k), as we could not detect a similar module in FTD-MAPT. In total, we observed 31 common putative miRNA targets in both networks.

Next, we selected hsa-miR-193a-3p, hsa-miR-150-5p and hsa-miR-19b-3p for further characterization in iPSC models (Fig. 6a). The first two miRNAs are DE in all three disease groups and have many targets among module genes (Fig. 5 j \& k). The miRNA hsa-miR$19 b-3 p$ is up-regulated in all disease groups, although it does not reach significance after (FTD-MAPT and FTD-C9orf72) or before (FTD-GRN) multiple testing correction. Nevertheless, down-regulated genes were predicted to be enriched for targets of hsa-miR$19 b-3 p$ by $g: P r o f i l e r$, the miRNA is known to inhibit autophagy ${ }^{31}$ and it is highly expressed in neurons. We performed RNA-seq on iPSC-derived neurons and microglia (Methods) that were transfected with miRNA mimics and inhibitors for the three selected miRNAs. Here, we focused on the mimic experiments, as the mimics should in theory reproduce the effects of miRNA overexpression. Inhibition and mimicking of miR-150-5p in neurons had only minor effects in neurons, while in microglia, the miR-150-5p mimic had strong effects, leading to 237 down-regulated and 236 up-regulated DEGs, enriched for cellular transport and immune system pathways of the latter and nervous system development of the former (Fig. 6b). Inhibition of miR-150-5p had even stronger effects (3221 DEGs), indicating an important function of this miRNA in microglia. Transfection of miR-193a-3p mimic and inhibitor only resulted in significant expression changes in microglia, where the mimic had strong effects with 1756 down-regulated and 1474 up-regulated genes. Up-regulated genes were enriched 
415 of mitochondrial functions like oxidative phosphorylation, while down-regulated genes were 416 enriched for localization and vesicle-mediated transport pathways (Fig. 6c).

417 In neurons, the miR-19b-3p mimic resulted in 89 down- and 137 up-regulated DEGs

418 (inhibitor: 8 down-regulated, 31 up-regulated). Genes down-regulated by the mimic and up419 regulated in the inhibitor experiment are involved in neuronal system pathways, enriched for 420 miR-19b-3p targets and share 17 common genes, thus providing evidence for these genes 421 to be regulated by miR-19b-3p. In microglia, stronger effects of the miR-19b-3p mimic 422 compared to the inhibitor were observed (1518 compared to 608 DEGs). Genes down423 regulated by the miR-19b-3p mimic were enriched for catabolic processes, autophagy and 424 vesicle-mediated transport, up-regulated genes were enriched for cell cycle and immune 425 system related genes (Fig. 6d). These results provide strong evidence that hsa-miR-19b-3p 426 and hsa-miR-193a-3p indeed regulate cellular trafficking pathways. Furthermore, hsa-miR$427 \quad 150-5 p$ is important for microglia function and up-regulation could lead to immune system 428 activation. 
a
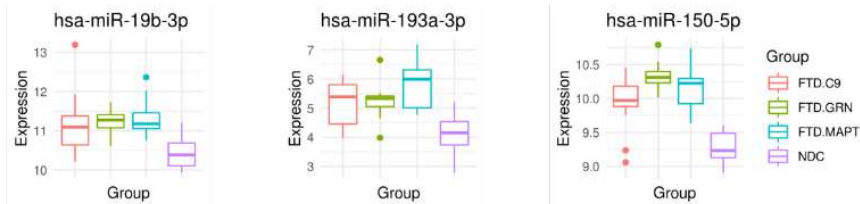

miR-150 Mimic Down

b

431

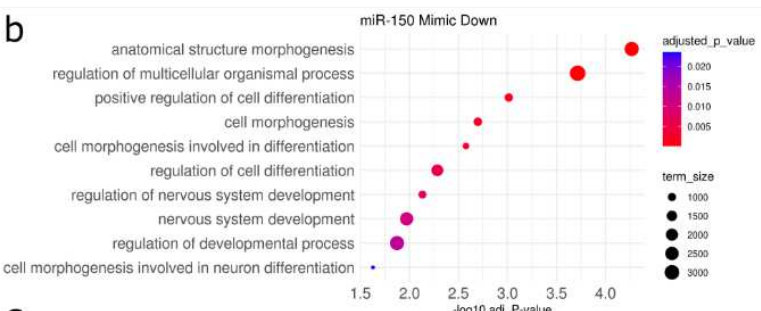

C

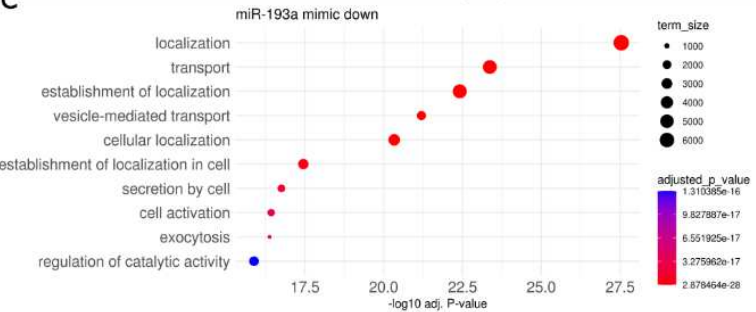

d
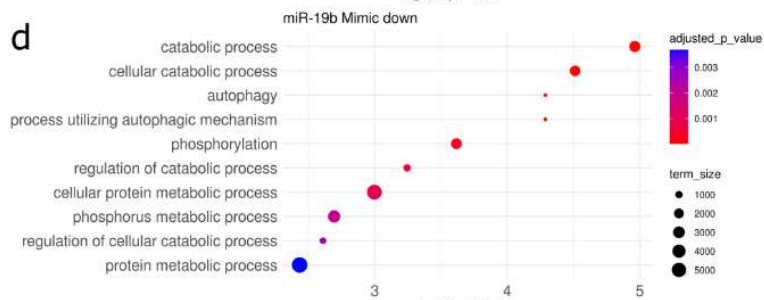

miR-150 Mimic Up

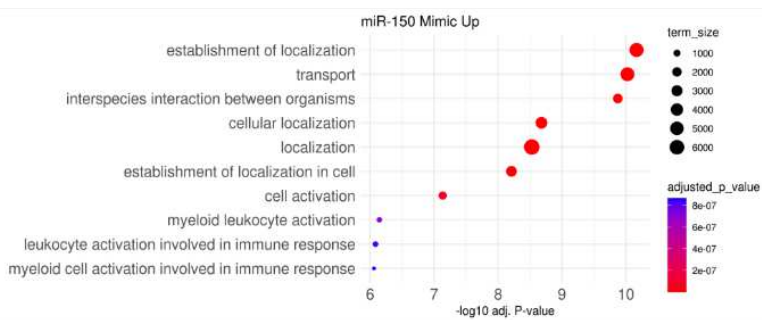

miR-193a mimic up
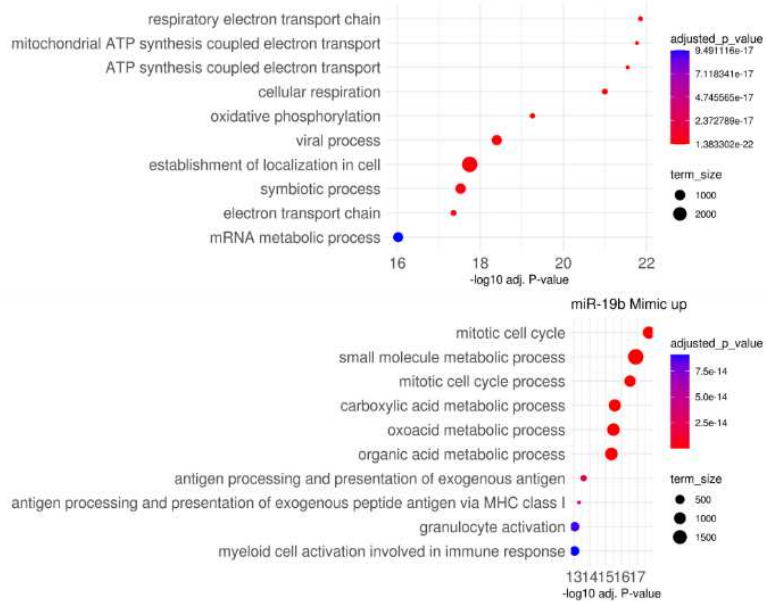

432 Figure 6: Effects of miRNA mimic and inhibitor experiments in iPSC-derived microglia.

433 a Boxplots of normalized expression values for the selected miRNAs. b, $\mathbf{c}, \mathbf{d}$ The top ten

434 most significantly enriched biological processes of up- and down-regulated genes after

435 transfection with mimics for miR-150-5p, miR-193a-3p and miR-19b-3p, respectively. Node

436 size corresponds to the number of genes in the biological process term and node colour

437 corresponds to the P-value adjusted for multiple testing.

441 Here, we present the data from the $1^{\text {st }}$ phase of the RiMod-FTD project, a multi-omics, multimodel data resource for FTD research that aims to understand the role of distinct genetic 
risk factors in the disease. Generated by the RiMod-FTD consortium over several years, the

444 resource depicts a valuable tool for FTD researchers that will help to accelerate scientific

445 progress towards a better understanding of relevant disease mechanisms in FTD. Additional 446 multi-omics data from iPSC derived cell types, transgenic mouse models and other brain 447 regions will be added over time.

449 By studying post-mortem tissue of the GFM we detected the largest transcriptional dysregulation and greatest neuronal loss in FTD caused by mutations in GRN, agreeing with previous findings of a more pronounced frontal lobe atrophy in FTD-GRN compared to other subtypes $^{32}$. Our deconvolution analysis indicates that excitatory neurons are the most affected cell type in all genetic subtypes of FTD, which was confirmed in the RiMod-FTD

454 proteomics data (Mediema et al., manuscript in preparation) as well. Recently, evidence from multiple studies has accumulated pointing toward a strong involvement of glutamatergic synapses in $\mathrm{FTD}^{33}$. While it has been previously reported that densities of both ionotropic glutamate receptors, AMPA and NMDA receptors, are reduced in post-mortem brain tissue of FTD patients ${ }^{21}$, we see evidence that AMPA receptors are particularly affected. Intriguingly, a recent single-nucleus RNA-seq study in a GRN mouse model could show that hyperactivation of microglia leads to selective loss of excitatory neurons ${ }^{34}$, which confirms our hypothesis that excitatory neurons are especially vulnerable in FTD.

Strong neuroinflammation is a distinct feature of FTD-GRN, which is confirmed in our data and by increased microglial cell numbers in this FTD sub-type, in line with recent findings showing increased microglial burden in FTD-GRN ${ }^{35,36}$. Using an integrative approach, we identified TFs: NFKB2, RELA, KLF3 and SP1 as key inflammatory drivers, leading to activation of the NFkB- and TNF-signalling pathways.

We found indicators of activated necroptosis, suggesting this pathway as potential cause for cell death. The necroptosis cell death pathway is deregulated in several neurodegenerative 
disorders $^{37}$, and a recent study has shown that TBK1, a genetic cause of ALS and FTD (here

470 down-regulated in FTD-GRN), is an endogenous inhibitor of RIPK1, an upstream regulator of

471 RIPK3 ${ }^{38}$. The authors showed that embryonic lethality of TBK1-knockout mice is dependent

472 on RIPK1 activity, suggesting that the necroptosis pathway is indeed an important player in

473 FTD pathogenesis. In a recent review, Molnár and colleagues have discussed several

474 available drugs that could potentially regulate necroptosis ${ }^{39}$, highlighting the potential of this

475 pathway as a drug target for developing therapies for FTD.

476

477 Our pathway enrichment and deconvolution analyses pointed toward increased blood vessel

478 abundance and growth in FTD brains compared to controls, which is consistent with the

479 results from the RiMod-FTD proteomics data (Mediema et al., manuscript in preparation). It

480 is generally not known how and if the vasculature system is involved in FTD pathogenesis,

481 although recent studies have observed abnormalities in a mouse model of tau pathology and

482 post-mortem human brains ${ }^{22,23}$. To our knowledge, angiogenesis as a pathological feature in

483 several genetic FTD subtypes has not been reported before and therefore depicts an

484 important subject for FTD research.

In all three disease groups, we have observed prominent up-regulation of ECM pathways and MMP enzymes, suggesting MMPs as important regulators in FTD pathogenesis. While it has been increasingly recognized that MMPs are important regulators in many neurodegenerative diseases ${ }^{40,41}$, the role of MMPs in FTD pathogenesis has not been investigated in depth. In mouse models of ALS, inhibition of the MMPs MMP2 and MMP9 could indeed prolong survival and reduce symptoms ${ }^{42,43}$. Moreover, TIMP3, which is upregulated in our data, was found to be partly responsible for neuronal apoptosis in an ALS model ${ }^{44}$, which points towards TIMP3 as a potential apoptosis mechanism in FTD. MMPs are furthermore tightly involved in the inflammatory response, and can activate the tumour 
necrosis factor (TNF) gene ${ }^{45}$. Inflammatory cytokines, hypoxia and reactive oxygen species can lead to the activation of MMPs ${ }^{40,46}$ and MMPs can digest the ECM, stimulate increased production of growth factors and thereby promote the growth of blood vessels, providing a potential causal link to the prominent enrichment of endothelial cells ${ }^{46}$. Given their important biological functions and their involvement in all genetic FTD subgroups, it will be important to further investigate how MMPs contribute to FTD and whether they can be exploited as drug targets, as MMP inhibition in model system has shown promising results ${ }^{47,48}$.

Impaired cellular trafficking mechanisms is very likely a key feature of FTD pathogenesis and it has been shown multiple times that FTD-causal mutations lead to trafficking deficits ${ }^{49-51}$. However, it is not always clear which mechanisms continue to dysfunctional transport mechanisms. Here, using multi-omics data and validation experiments, we show that elevated expression of several miRNAs contributes to the inhibition of genes important for cellular transport. Additional studies are necessary to further validate this hypothesis, which directly suggests several miRNAs as putative drug targets.

511 While our study is reasonably powered, increasing the sample size for individual groups such as FTD-C9orf72 would further increase the power of our analysis and help to better define which pathways are truly distinct to certain subtypes. It will therefore be an objective

514 for future iterations of the RiMod-FTD resource to include larger numbers of samples.

516 To conclude, we present here an integrated multi-omics analysis on data from Phase 1 of

517 the RiMod-FTD project and developed new hypotheses on FTD disease mechanisms. The 518 data presented here highlights several regulator molecules important for FTD pathogenesis 519 and their consequences such as vascular abnormalities and thereby we show the value of an integrated multi-omics data analysis for hypothesis generation and testing. The RiMod- 
521 FTD data will be freely accessible to the scientific community through the European

522 Genome-phenome Archive (EGA) and a dedicated RiMod-FTD web application

523 (https://www.rimod-ftd.org) thus enabling scientists to derive new mechanisms and

524 hypotheses from the data.

525

526

527

528

529

530 Methods

531 Donor samples employed in this study

532 Post mortem human brains

533 Tissues were obtained under a Material Transfer Agreement from the Netherlands Brain

534 Bank, and additional samples were provided by the Queen Square Brain Bank of

535 Neurological Disorders and MRC, King College London. Demographic details about human

536 brain samples are summarized in Table S1.

537 GFM and GTM tissue from each subject was divided into three pieces for transcriptomic,

538 proteomic and epigenetic experiments in a dry-ice bath using precooled scalpels and

539 plasticware.

540

541 hIPS-derived NGN2 neurons and miRNA mimics and inhibitors transfection

542 smNPC were derived from hiPSc cells (Cell line id: GM23280 obtained from the Coriell

543 Institute) using the protocol described by Reinhardt et al ${ }^{52}$. The differentiation protocol from 
544 smNPC to neurons involves over-expression of Neurogenin-2 (NGN2) using a modified

545 version of the NGN2 lentiviral inducible vector system (single vector

546 pLV_TRET_hNgn2_UBC_BSD_T2A_rtTA3). The detailed description about protocol,

547 reagents and media composition is available in Dhingra et al. ${ }^{53}$.

548 Briefly, stable NGN2 smNPC are grown for six days in expansion medium N2B27

549 supplemented with CHIR99021 (CHIR) $3 \mu \mathrm{M}$, Purmorphamine (PMA) $0.5 \mu \mathrm{M}$ and L-ascorbic

550 acid 2-phosphate magnesium (AA) $64 \mathrm{mg} / \mathrm{l}$. For differentiation, cells are plated $(80,000$

551 cells/cm2) onto Poly L-orithine and laminin coated plates in N2B27 medium supplemented

552 with doxycycline (dox) at $2.5 \mu \mathrm{g} / \mathrm{mL}$, and $2 \mu \mathrm{M}$ DAPT. On day 4 of differentiation,

553 transfection was performed in $n=3$ replicate plates using lipofectamine RNAiMax

554 (ThermoFisher Scientific) with a final concentration of miRNA mimic and inhibitors (miR-19b-

$5553 p$ and miR-1505p mimics and inhibitors from Qiagen and miR-193a-3p mimic and inhibitor

556 from ThermoFisher Scientific) in the range of 5 to $10 \mathrm{nM}$ as per the manufactures' guidelines

557 along with their corresponding controls. Next day (day 5 of differentiation), the complete

558 media was changed with N2B27 media supplemented with dox, $10 \mathrm{ng} / \mathrm{mL}$ brain-derived

559 neurotrophic factor (BDNF), $10 \mathrm{ng} / \mathrm{mL}$ glial cell-derived neurotrophic factor (GDNF), 10

$560 \mathrm{ng} / \mathrm{mL}$ neurotrophic factor 3 (NT-3), $1 \mu \mathrm{g} / \mathrm{mL}$ Laminin, and $10 \mu \mathrm{M}$ DAPT. Thereafter, half

561 media was changed on day 8 of differentiation. On day 11, cells were gently washed with

562 PBS and processed for RNA isolation.

563

hIPS-derived microglia and miRNA mimics and inhibitors transfection

hiPSCs were differentiated as previously described (van Wilgenburg et $\mathrm{al}^{54}$ ). In brief, $3 \mathrm{x}$ $10^{\wedge} 6$ iPSCs were seeded into an Aggrewell 800 well (STEMCELL Technologies) to form embryoid bodies (EBs), in mTeSR1 and fed daily with medium plus 50ng/ml BMP4 (Miltenyi

568 Biotec), 50ng/ml VEGF (Miltenyi Biotec), and 20ng/ml SCF (R\&D Systems). Four-day EBs

569 were then differentiated in 6-well plates (15 EBs/well) in X-VIVO15 (Lonza) supplemented 
570 with 100ng/ml M-CSF (Miltenyi Biotec), 25ng/ml IL-3 (Miltenyi Biotec), 2mM Glutamax

571 (Invitrogen Life Technologies), and $0.055 \mathrm{mM}$ beta-mercaptoethanol (Thermo Fisher

572 Scientific), with fresh medium added weekly. Microglial precursors emerging in the

573 supernatant after approximately 1 month were collected and isolated through a 40um cell

574 strainer and plated in N2B27 media supplemented with $100 \mathrm{ng} / \mathrm{ml} \mathrm{M-CSF,} 25 \mathrm{ng} / \mathrm{ml}$

575 interleukin 34 (IL-34) for differentiation. Thereafter, the media is reshred every 2 days

576 supplemented with $100 \mathrm{ng} / \mathrm{ml} \mathrm{M-CSF}$, and $25 \mathrm{ng} / \mathrm{ml} \mathrm{IL-34.} \mathrm{The} \mathrm{cells} \mathrm{were} \mathrm{cultured} \mathrm{for}$

577 additional 6 days with media refresh every 2 days. On day 7 of maturation, transfection was

578 performed in $\mathrm{n}=3$ replicate plates using lipofectamine RNAiMax with a final concentration of

579 miRNA mimics and inhibitors in the range of 5 to $10 \mathrm{nM}$ as per the manufactures' guidelines

580 along with their corresponding controls (miR-19b-3p and miR-1505p mimics and inhibitors

581 from Qiagen and miR-193a-3p mimic and inhibitor from ThermoFisher Scientific). Next day

582 complete media was refreshed. On day 11, cells were gently washed with PBS and

583 processed for RNA isolation.

584

585 Genetic analysis

586 Genomic DNA was isolated from $50 \mathrm{mg}$ of GFM frozen brain tissue by using the Qiamp DNA

587 mini kit (Qiagen) following the manufacturer protocol. DNA concentration and purity were

588 assessed by nanodrop measurement. DNA integrity was evaluated by loading 100

589 nanogram per sample on a $0,8 \%$ agarose gel and comparing size distribution to a size

590 standard.

591 Presence of C9orf72-HRE in postmortem brain tissues and hIPS cells was confirmed by

592 primed repeat PCR according to established protocols. Reported mutations for MAPT and

593 GRN were verified by sanger sequencing. 
597 Total RNA for CAGE-seq and RNAseq was isolated from $\pm 100 \mathrm{mg}$ of frozen brain tissue with

598 TRIzol reagent (Thermo Fischer Scientific) according to the manufacturer recommendation, 599 followed by purification with the RNeasy mini columns (Qiagen) after DNAse treatment.

600

601 Total RNA for smallRNA-seq was isolated from frozen tissue using the TRIzol reagent

602 (ThermoFischer Scientific). After isopropanol precipitation and $80 \%$ ethanol rinsing RNA

603 pellet was resuspended in RNAse free water and up to 10 micrograms of RNA was

604 incubated with $2 \mathrm{U}$ of Ambion DNAse I (ThermoFischer) at $37^{\circ} \mathrm{C}$ for 20 minutes. DNA-free

605 RNA samples were then further purified by phenol-chloroform-isoamyl-alchol extraction

606 followed by ethanol precipitation.

607

608

RNA isolation from smNPC-derived neurons and microglia

609

Total RNA was isolated from NGN2 driven neurons and microglia cells after transfection with

610 miRNA mimics and inhibitors. Briefly at day 11 of transfection cells were carefully rinsed with

611 PBS and lysed in Qiazol buffer (Qiagen). Further DNAse treatment and purification were

612 carried out with the miRNeasy micro kit (Qiagen) according to the manufacturer protocol.

613

614 RNA QC

615 For each RNA sample, RNA concentration $\left(A_{260}\right)$ and purity $\left(A_{260 / 280}\right.$ and $\left.A 2_{60 / 230}\right)$ were

616 determined by Nanodrop measurement and RNA integrity (RIN) was assessed on a

617 Bioanalyser 2100 system and/or Tape station 41200 (Agilent Technologies Inc.)

618

619 CAGE-seq libraries 
620 CAGE-seq libraries were prepared from 5 micrograms of RNA from frozen brain tissues

621 according to a published protocol ${ }^{55}$. Libraries were sequenced on a HiSeq 2000 and/or

622 HiSeq2500 on a $1 \times 50$ bp single read (SR) flow cell (Illumina) at an average of 20M

623 reads/sample.

624

$625 \quad$ RNAseq libraries

626 Total RNAseq libraries were prepared from 1 microgram of total RNA from frozen brain

627 tissue using the TruSeq Stranded Total RNA with Ribo-Zero Gold kit (Illumina) according to

628 the protocol specifications. RNAseq libraries were sequenced on a Hiseq2500 and

629 HISeq4000 on a $2 \times 100$ bp paired end (PE) flow cell (Illumina) at an average of $100 \mathrm{M}$

630 PE/sample.

631

632

smallRNAseq libraries

633 Small RNA-seq libraries were prepared from 1 microgram of total RNA from NPC-derived neurons and 300 nanograms of microglia after miRNA mimics and inhibitors transfection, using the mRNA TrueSeq Stranded kit (Illumina). mRNAseq libraries were sequenced on a

636 NextGen550 on a 75 cycles flow cell (Illumina). Small RNAseq libraries from frozen tissue were prepared starting from 2 micrograms of total RNA using the Nextflex Small RNA-seq kit v3 (Bioo Scientific) and the NEBNext Small RNA library prep set for Illumina (New England Biolabs). Libraries were sequenced on a NextSeq550 on a 75 cycles flow cell.

640

641

642 Methylation assay 
643 To assess the methylation status of over $850000 \mathrm{CpG}$ sites in promoter, gene body and

644 enhancer regions we have used the MethylationEPIC bead chip arrays (Illumina).

645 Bisulfite conversion of genomic DNA, genome amplification, hybridization to the beadchips, 646 washing, staining and scanning procedure was performed by Atlas Biolabs (Atlas Biolabs,

647 Berlin, Germany). Cases and controls DNAs were distributed randomly across each array.

648

649 HumanBase Module Analysis

650 Functional gene modules were generated using the HumanBase tool at:

651 https://hb.flatironinstitute.org/. We divided DEGs into up- and down-regulated genes as we were looking for active and repressed modules in FTD. Modules were downloaded for further analysis. Cell type enrichment analysis was performed for genes of each modules using

654 EWCE $^{56}$ as described further down.

\section{RNA-seq processing and analysis}

656 Raw FastQ files were processed using the RNA-seq pipeline from nf-core (nf-core/rnaseq v1.3 ${ }^{57}$, with trimming enabled. Gene quantification was subsequently done using Salmon (v0.14.1 $)^{58}$ on the trimmed FastQ files. Alignment and mapping were performed against the human genome hg38. DESeq2 (v.1.26.0) ${ }^{59}$ was used to perform differential expression analysis. We corrected for the covariates gender and PH-value. Genes were considered differentially expressed when having a Benjamini-Hochberg corrected P-value below 0.05.

\section{Cell type deconvolution and filtering}

We performed cell type deconvolution on the RNA-seq data using Scaden ${ }^{60}$. For training we used the human brain training dataset used in the Scaden publication. Each ensembl model was trained for 5000 steps. To filter differentially expressed genes for false positives caused by cell composition bias, we first calculated the correlation of gene expression with cell type

667 fraction. Then, we calculated a cell type specificity score as defined in Skene et al. ${ }^{56}$ for 
each gene available in the scRNA-seq dataset from Darmanis et al. ${ }^{61}$. We filtered out all

669 genes that had a specificity score of at least 0.5 and a positive correlation of at least 0.4 with

670 the cell type fractions of the most specific cell type. False positive DEGs that are caused by

671 systematic increase or decrease of a specific cell type will show high correlation with the cell

672 type fractions and can thus be identified and removed from the analysis. A specificity score

673 of 0.5 means that half of the total gene expression for a certain gene can be attributed to a

674 single cell type, assuming a uniform cell type composition. The cut-offs for specificity score

675 and correlation were chosen based on an informed decision. Relative changes in cell type

676 composition were quantified by first calculating the average fractions of a cell type for all

677 groups and then calculating the percentual change of cell fractions compared to the average

678 control fractions. This allows to detect relative changes in cell type compositions. Statistical

679 significance between cell type fractions of groups was assessed using a t-test in the $R$

680 language.

681 Cell type enrichment analysis

682 We performed cell type enrichment analysis of genesets using the EWCE R package ${ }^{56}$. Cell

683 type specificity of genes was calculated from the single-cell RNA-seq cortex dataset of

684 Darmanis and colleagues ${ }^{61}$. EWCE analysis was done following instructions from

685 https://github.com/NathanSkene/EWCE.

686 CAGE-seq processing and analysis

687 Sequencing adapters and barcodes in CAGE-seq FastQ files were trimmed using Skewer

688 (v.0.1.126) ${ }^{62}$. Sequencing artefacts were removed using TagDust (v1.0) ${ }^{63}$. Processed reads 689 were then aligned against the human genome hg38 using STAR (v.2.4.1 $)^{64}$. CAGE detected 690 TSS (CTSS) files were created using CAGEr (v1.10.0 $)^{65}$. With CAGEr, we removed the first

691 G nucleotide if it was a mismatch. CTSS were clustered using the 'distclu' method with a 692 maximum distance of $20 \mathrm{bp}$. For exact commands used we refer to the reader to the scripts 693 used in this pipeline: https://github.com/dznetubingen/cageseq-pipeline-mf. 
695 To identify candidate regulatory transcription factors, we first performed differential 696 expression analysis with all CAGE-seq clusters (see RNA-seq analysis). Then, we extracted 697 the sequence $600 \mathrm{bp}$ up-stream and $300 \mathrm{bp}$ downstream around all detected clusters. We 698 used Homer ${ }^{66}$ to look for significant TFBS enrichment in the regions around up- and downregulated clusters (similar to ${ }^{67}$ ). TFBS motifs were downloaded from the JASPAR database ${ }^{68}$. When calculating enrichment, we considered all extracted regions that are not part of the set of interest as background. The complete pipeline can be found at https://github.com/KevinMenden/tf-activity. We selected all TFs with significant enrichment ( $p$-value $<=0.001$ ) for either up-regulated or down-regulated CAGE clusters as candidate regulators. We considered genes as potential targets of a TF if a TFBS could be found in their promoter region. As an additional filter, we selected only TFs with evidence for differential expression in the RNA-seq data (adj. P-value $<0.05$, not filtered for cell composition).

\section{smRNA-seq processing and analysis}

709

After removing sequencing adapters, all FastQ files were uploaded to OASIS2 ${ }^{69}$ for analysis. Subsequent differential expression analysis was performed on the counts yielded from OASIS2, using DESeq2 and correcting for gender and $\mathrm{PH}$-value, as was done for the

712 RNA-seq data. Additionally, we added a batch variable to the design matrix to correct for the

713 two different batches of this dataset. For the target prediction analysis, we first downloaded

714 all targets from mirBase ${ }^{70}$. Then, we correlated the expression of miRNAs with their

715 predicted targets using matching samples from the RNA-seq data. We removed all predicted

716 targets with a correlation above -0.4 , thus only considering miRNA-target pairings with high 717 negative correlation.

\section{Methylation data processing and analysis}

720 The Infinium MethylationEPIC BeadChip data was analyzed using the minfi R package ${ }^{71}$. We 
removed all sites with a detection P-value above 0.01 , on sex chromosomes and with single

722 nucleotide polymorphisms (SNPs). Data normalization was done using stratified quantile

723 normalization. Sites with a standard deviation below 0.1 were considered uninformative and

724 filtered out, to increase detection power. Surrogate variable analysis ${ }^{72}$ was performed to

725 determine confounding factors. Differential methylation analysis was done using the limma

726 package ${ }^{73}$ and controlling for the detected surrogate variables. Sites with a Benjamini-

727 Hochberg ${ }^{74}$ adjusted P-value below 0.05 were considered differentially methylated.

\section{Age prediction}

729 We predicted the biological age of donors using the methylation data and the Wenda

730 algorithm ${ }^{75}$. Training data was kindly provided by the authors of Wenda. We subsetted the

731 data for CpG sites found in our data $(11,729)$ sites and performed the prediction as

732 described at https://github.com/PfeiferLabTue/wenda.

733

\section{Analysis of mRNA-seq data from cellular models}

This section describes the analysis of mRNA-seq data generated for the miRNA mimic and

736 inhibitor experiments. FastQ files were mapped and gene counts quantified using Salmon and differential expression analysis was performed with DESeq2 (see post-mortem brain

738 RNA-seq analysis). DEGs were examined for pathway enrichment using go:Profiler.

\section{Assessment of degeneration}

741 For assessment of neurodegeneration, H\&E stained paraffin sections of the frontal and

742 temporal cortex were graded as absent (0), mild (1), moderate (2) and severe (3) based on 743 the presence of spongiosis, neuronal loss and gliosis. 
746 All data used in this study and published as phase 1 of the RiMod-FTD resource have been

747 deposited at the European Phenome-genome Archive (EGA) under accession number

\section{Code Availability}

The code used for generating the analysis results is made freely available in the GitHub repository https://github.com/dznetubingen/rimod-ftd-paper.

\section{References}

753

754

755

756

757

758

759

760

761

762

763

764

765

766

767

768

769

770

771

772

773

774

775

776

777

1. Bang, J., Spina, S. \& Miller, B. L. Frontotemporal dementia. Lancet 386, 1672-1682 (2015).

2. Panza, F. et al. Development of disease-modifying drugs for frontotemporal dementia spectrum disorders. Nat. Rev. Neurol. 16, 213-228 (2020).

3. Seelaar, H., Rohrer, J. D., Pijnenburg, Y. A. L., Fox, N. C. \& van Swieten, J. C. Clinical, genetic and pathological heterogeneity of frontotemporal dementia: a review. J. Neurol. Neurosurg. Psychiatry 82, 476-486 (2011).

4. Olszewska, D. A., Lonergan, R., Fallon, E. M. \& Lynch, T. Genetics of Frontotemporal Dementia. Current Neurology and Neuroscience Reports vol. 16 (2016).

5. Sirkis, D. W., Geier, E. G., Bonham, L. W., Karch, C. M. \& Yokoyama, J. S. Recent Advances in the Genetics of Frontotemporal Dementia. Curr. Genet. Med. Rep. 7, 4152 (2019).

6. Sieben, A. et al. The genetics and neuropathology of frontotemporal lobar degeneration. Acta Neuropathologica vol. 124 353-372 (2012).

7. Balendra, R. \& Isaacs, A. M. C9orf72-mediated ALS and FTD: multiple pathways to disease. Nat. Rev. Neurol. 1 (2018) doi:10.1038/s41582-018-0047-2.

8. RiMod-FTD | JPND. https://www.neurodegenerationresearch.eu/initiatives/annualcalls-for-proposals/closed-calls/risk-factors-2012/risk-factor-call-results/rimod-ftd/.

9. Raudvere, U. et al. g:Profiler: a web server for functional enrichment analysis and conversions of gene lists (2019 update). Nucleic Acids Res. 47, W191-W198 (2019).

10. Golpich, M. et al. Mitochondrial Dysfunction and Biogenesis in Neurodegenerative diseases: Pathogenesis and Treatment. CNS Neurosci. Ther. 23, 5-22 (2017).

11. Li, Y., Li, Z.-X., Jin, T., Wang, Z.-Y. \& Zhao, P. Tau Pathology Promotes the Reorganization of the Extracellular Matrix and Inhibits the Formation of Perineuronal Nets by Regulating the Expression and the Distribution of Hyaluronic Acid Synthases. 
12. Végh, M. J. et al. Reducing hippocampal extracellular matrix reverses early memory deficits in a mouse model of Alzheimer's disease. Acta Neuropathol. Commun. 2, (2014).

13. Lu, P., Takai, K., Weaver, V. M. \& Werb, Z. Extracellular Matrix degradation and remodeling in development and disease. Cold Spring Harb. Perspect. Biol. 3, (2011).

14. Duits, F. H. et al. Matrix Metalloproteinases in Alzheimer's Disease and Concurrent

15. Xi, Z. et al. Hypermethylation of the CpG Island Near the G 4 C 2 Repeat in ALS with a C9orf72 Expansion. Am J Hum Genet 981-989 (2013) doi:10.1016/j.ajhg.2013.04.017.

16. Biswas, M. H. U. et al. MMP-9 and MMP-2 Contribute to Neuronal Cell Death in iPSC Models of Frontotemporal Dementia with MAPT Mutations. Stem Cell Reports 7, 316324 (2016).

17. Young, J. J., Lavakumar, M., Tampi, D., Balachandran, S. \& Tampi, R. R. Frontotemporal dementia: latest evidence and clinical implications. Ther. Adv. Psychopharmacol. 8, 33-48 (2018).

18. Cash, D. M. et al. Patterns of gray matter atrophy in genetic frontotemporal dementia: results from the GENFI study. Neurobiol. Aging 62, 191-196 (2018).

19. Fu, H., Hardy, J. \& Duff, K. E. Selective vulnerability in neurodegenerative diseases. Nat. Neurosci. 21, 1350-1358 (2018).

20. Palese, F. et al. Anti-GluA3 antibodies in frontotemporal dementia: effects on glutamatergic neurotransmission and synaptic failure. Neurobiol. Aging 86, 143-155 (2020).

21. Murley, A. G. \& Rowe, J. B. Neurotransmitter deficits from fronto temporal lobar degeneration. Brain 141, 1263-1285 (2018).

22. Bennett, R. E. et al. Tau induces blood vessel abnormalities and angiogenesis-related gene expression in P301L transgenic mice and human Alzheimer's disease. Proc. Natl. Acad. Sci. U. S. A. 115, E1289-E1298 (2018).

23. Ek Olofsson, H. \& Englund, E. A cortical microvascular structure in vascular dementia, Alzheimer's disease, frontotemporal lobar degeneration and nondemented controls: a sign of angiogenesis due to brain ischaemia? Neuropathol. Appl. Neurobiol. 45, 557569 (2019).

24. Park, L. et al. Tau induces PSD95-neuronal NOS uncoupling and neurovascular dysfunction independent of neurodegeneration. Nat. Neurosci. 23, 1079-1089 (2020).

25. Greene, C. S. et al. Understanding multicellular function and disease with human tissue-specific networks. Nat. Genet. 47, 569-576 (2015).

26. Skene, N. G. \& Grant, S. G. N. Identification of Vulnerable Cell Types in Major Brain Disorders Using Single Cell Transcriptomes and Expression Weighted Cell Type Enrichment. Front. Neurosci. 10, 16 (2016).

27. Paushter, D. H., Du, H., Feng, T. \& Hu, F. The lysosomal function of progranulin, a guardian against neurodegeneration. Acta Neuropathol. 136, 1 (2018).

28. Lui, H. et al. Progranulin Deficiency Promotes Circuit-Specific Synaptic Pruning by Microglia via Complement Activation. Cell 165, 921-935 (2016). 
29. Krabbe, G. et al. Microglial NFKB-TNFa hyperactivation induces obsessivecompulsive behavior in mouse models of progranulin-deficient Frontotemporal dementia. Proc. Natl. Acad. Sci. U. S. A. 114, 5029-5034 (2017).

30. Martens, L. H. et al. Progranulin deficiency promotes neuroinflammation and neuron loss following toxin-induced injury. J. Clin. Invest. 122, 3955-3959 (2012).

31. Zou, M. et al. Autophagy inhibition of hsa-miR-19a-3p/19b-3p by targeting TGF- $\beta$ R II during TGF- $\beta 1$-induced fibrogenesis in human cardiac fibroblasts. Sci. Rep. 6, 1-15 (2016).

32. Whitwell, J. L. et al. Neuroimaging signatures of frontotemporal dementia genetics: C9ORF72, tau, progranulin and sporadics. Brain 135, 794-806 (2012).

33. Benussi, A. et al. Toward a glutamate hypothesis of frontotemporal dementia. Front. Neurosci. 13, (2019).

34. Zhang, J. et al. Neurotoxic microglia promote TDP-43 proteinopathy in progranulin deficiency. Nature (2020) doi:10.1038/s41586-020-2709-7.

35. Woollacott, I. O. C. \& Rohrer, J. D. The clinical spectrum of sporadic and familial forms of frontotemporal dementia. J. Neurochem. 138, 6-31 (2016).

36. Sakae, N. et al. Microglia in frontotemporal lobar degeneration with progranulin or C9ORF72 mutations. Ann. Clin. Transl. Neurol. 6, 1782-1796 (2019).

37. Yuan, J., Amin, P. \& Ofengeim, D. Necroptosis and RIPK1-mediated neuroinflammation in CNS diseases. Nat. Rev. Neurosci. 20, 19-33 (2019).

38. Xu, D. et al. TBK1 Suppresses RIPK1-Driven Apoptosis and Inflammation during Development and in Aging. Cell 174, 1477-1491.e19 (2018).

39. Molnár, T. et al. Current translational potential and underlying molecular mechanisms of necroptosis. Cell Death Dis. 10, 1-21 (2019).

40. Brkic, M., Balusu, S., Libert, C. \& Vandenbroucke, R. E. Friends or Foes: Matrix Metalloproteinases and Their Multifaceted Roles in Neurodegenerative Diseases. Mediators Inflamm. 2015, (2015).

41. Rivera, S., García-González, L., Khrestchatisky, M. \& Baranger, K. Metalloproteinases and their tissue inhibitors in Alzheimer's disease and other neurodegenerative disorders. Cell. Mol. Life Sci. 76, 3167-3191 (2019).

42. Kiaei, M. et al. Matrix metalloproteinase- 9 regulates TNF- $\alpha$ and FasL expression in neuronal, glial cells and its absence extends life in a transgenic mouse model of amyotrophic lateral sclerosis. Exp. Neurol. 205, 74-81 (2007).

43. Lorenzl, S. et al. The matrix metalloproteinases inhibitor Ro 26-2853 extends survival in transgenic ALS mice. Exp. Neurol. 200, 166-171 (2006).

44. Lee, J. K. et al. Tissue inhibitor of metalloproteinases-3 (TIMP-3) expression is increased during serum deprivation-induced neuronal apoptosis in vitro and in the G93A mouse model of amyotrophic lateral sclerosis: A potential modulator of Fasmediated apoptosis. Neurobiol. Dis. 30, 174-185 (2008).

45. Vandenbroucke, R. E. et al. Matrix metalloproteinase 13 modulates intestinal epithelial barrier integrity in inflammatory diseases by activating TNF. EMBO Mol. Med. 5, 1000-1016 (2013).

46. Rempe, R. G., Hartz, A. M. S. \& Bauer, B. Matrix metalloproteinases in the brain and blood-brain barrier: Versatile breakers and makers. J. Cereb. Blood Flow Metab. 36, 
47. Woo, M. S., Park, J. S., Choi, I. Y., Kimf, W. K. \& Kim, H. S. Inhibition of MMP-3 or -9 suppresses lipopolysaccharide-induced expression of proinflammatory cytokines and iNOS in microglia. J. Neurochem. 106, 770-780 (2008).

48. Garcia-Alloza, M. et al. Matrix metalloproteinase inhibition reduces oxidative stress associated with cerebral amyloid angiopathy in vivo in transgenic mice. J. Neurochem. 109, 1636-1647 (2009).

872

873

874

49. Aoki, Y. et al. C9orf72 and RAB7L1 regulate vesicle trafficking in amyotrophic lateral sclerosis and frontotemporal dementia. Brain 887-897 (2017) doi:10.1093/brain/awx024.

876

877

878

879

880

881

882

883

884

885

886

887

888

889

890

891

892

893

894

895

896

897

898

899

900

901

902

903

904

905

906

50. Yarwood, R., Hellicar, J., Woodman, P. G. \& Lowe, M. Membrane trafficking in health and disease. DMM Dis. Model. Mech. 13, (2020).

51. Wren, M. C. et al. Frontotemporal dementia-associated N279K tau mutant disrupts subcellular vesicle trafficking and induces cellular stress in iPSC-derived neural stem cells. Mol. Neurodegener. 10, 46 (2015).

52. Reinhardt, P., Glatza, M., Hemmer, K., Tsytsyura, Y. \& Thiel, C. S. Derivation and Expansion Using Only Small Molecules of Human Neural Progenitors for Neurodegenerative Disease Modeling. PLoS One 8, 59252 (2013).

53. Dhingra, A. et al. Automated production of human induced pluripotent stem cellderived cortical and dopaminergic neurons with integrated live-cell monitoring. J. Vis. Exp. 2020, 1-29 (2020).

54. van Wilgenburg, B., Browne, C., Vowles, J. \& Cowley, S. A. Efficient, Long Term Production of Monocyte-Derived Macrophages from Human Pluripotent Stem Cells under Partly-Defined and Fully-Defined Conditions. PLoS One 8, (2013).

55. Takahashi, H., Lassmann, T., Murata, M. \& Carninci, P. 5' end-centered expression profiling using cap-analysis gene expression and next-generation sequencing. Nat. Protoc. 7, 542-561 (2012).

56. Skene, N. G. \& Grant, S. G. N. Identification of Vulnerable Cell Types in Major Brain Disorders Using Single Cell Transcriptomes and Expression Weighted Cell Type Enrichment. Front. Neurosci. 10, 16 (2016).

57. Ewels, P., Magnusson, M., Lundin, S. \& Käller, M. MultiQC: Summarize analysis results for multiple tools and samples in a single report. Bioinformatics 32, 3047-3048 (2016).

58. Patro, R., Duggal, G., Love, M. I., Irizarry, R. A. \& Kingsford, C. Salmon provides fast and bias-aware quantification of transcript expression. Nat. Publ. Gr. 14, (2017).

59. Love, M. I., Huber, W. \& Anders, S. Moderated estimation of fold change and dispersion for RNA-seq data with DESeq2. Genome Biol. 15, (2014).

60. Menden, K. et al. Deep learning-based cell composition analysis from tissue expression profiles. Sci. Adv. 6, eaba2619 (2020).

61. Darmanis, S. et al. A survey of human brain transcriptome diversity at the single cell level. Proc. Natl. Acad. Sci. U. S. A. 112, 7285-7290 (2015).

62. Jiang, H., Lei, R., Ding, S. W. \& Zhu, S. Skewer: A fast and accurate adapter trimmer for next-generation sequencing paired-end reads. BMC Bioinformatics 15, 182 (2014).

909 63. Lassmann, T. TagDust2: A generic method to extract reads from sequencing data. 
BMC Bioinformatics 16, 24 (2015).

911

912

913

914

915

916

917

918

919

920

921

922

923

924

925

926

927

928

929

930

931

932

933

934

935

936

937

938

939

940

941

942

943

64. Dobin, A. \& Gingeras, T. R. Mapping RNA-seq Reads with STAR. in Current Protocols in Bioinformatics (2015). doi:10.1002/0471250953.bi1114s51.

65. Haberle, V., Forrest, A. R. R., Hayashizaki, Y., Carninci, P. \& Lenhard, B. CAGEr: Precise TSS data retrieval and high-resolution promoterome mining for integrative analyses. Nucleic Acids Res. 43, e51-e51 (2015).

66. Heinz, S. et al. Simple Combinations of Lineage-Determining Transcription Factors Prime cis-Regulatory Elements Required for Macrophage and B Cell Identities. Mol. Cell 38, 576-589 (2010).

67. Arenillas, D. J. et al. CAGEd-oPOSSUM: motif enrichment analysis from CAGEderived TSSs. Bioinformatics 32, 2858-60 (2016).

68. Khan, A. et al. JASPAR 2018: update of the open-access database of transcription factor binding profiles and its web framework. Nucleic Acids Res. 46, D260-D266 (2018).

69. Rahman, R. U. et al. Oasis 2: Improved online analysis of small RNA-seq data. BMC Bioinformatics 19, 54 (2018).

70. Kozomara, A., Birgaoanu, M. \& Griffiths-Jones, S. MiRBase: From microRNA sequences to function. Nucleic Acids Res. 47, D155-D162 (2019).

71. Aryee, M. J. et al. Minfi: A flexible and comprehensive Bioconductor package for the analysis of Infinium DNA methylation microarrays. Bioinformatics 30, 1363-1369 (2014).

72. Leek, J. T. \& Storey, J. D. Capturing Heterogeneity in Gene Expression Studies by Surrogate Variable Analysis. PLoS Genet. 3, e161 (2007).

73. Ritchie, M. E. et al. limma powers differential expression analyses for RNAsequencing and microarray studies. Nucleic Acids Res. (2015) doi:10.1093/nar/gkv007.

74. Benjamini, Y. \& Hochberg, Y. Controling the False Discovery Rate: A Practical and Powerful Approach to Multiple Testing. J. R. Stat. Soc. (1995).

75. Handl, L., Jalali, A., Scherer, M., Eggeling, R. \& Pfeifer, N. Weighted elastic net for unsupervised domain adaptation with application to age prediction from DNA methylation data. in Bioinformatics vol. 35 i154-i163 (Oxford University Press, 2019). 


\section{Acknowledgements}

946 Post-mortem brain tissue was obtained from the Dutch Brain Bank, Netherlands Institute for

947 Neuroscience, Amsterdam, and from the London Neurodegenerative Disease Brain Bank,

948 King's College London, London, UK. The London Neurodegenerative Disease Brain Bank is 949 part of the Brains for Dementia Research Initiative.

950 Author contributions

951 The project was initiated and designed by PH. Small RNA-seq experiments were performed

952 by AF, LK, PR and NF. RNA-seq and CAGE-seq experiments were performed by CB, PR,

$953 \mathrm{NF}, \mathrm{MC}$. iPSC-derived neuron and microglia experiments were performed by AD and DKV.

954 Analysis of CAGE-seq data was done by MF, TN and KM. JSS, BA and KM analysed the

955 RNA-seq data. KM analysed the smRNA-seq and methylation data. PH, SB, PR planned

956 and interpreted all performed analyses. KM, PH, PR and SB wrote the manuscript.

\section{$957 \quad$ Funding}

958 This work was funded in part by the EU Joint Programme - Neurodegenerative Disease

959 Research (JPND) project: Risk and Modifying factors for FTD (RiMod-FTD) and the NOMIS

960 Foundation. DKV is in receipt of an Alexander von Humboldt Research Fellowship.

\section{RiMod-FTD Partners}

962 Prof. Dr. P. Heutink: Kevin Menden; Margherita Francescatto; Tenzin Nyima; Cornelis

963 Blauwendraat; Burcu Atarsu; Javier Simon Sanchez; Stefan Bonn; Patrizia Rizzu;

964 German Center for Neurodegenerative Diseases (DZNE)-Tübingen 
967 Germany

968 Tel.: +49(0)70719254050

969 E-Mail: peter.heutink@dzne.de

970

971 Prof. Dr. J. Hardy:

972 Reta Lila Weston Research Laboratories, Dept. of

973 Molecular Neuroscience, UCL Institute of

974 Neurology, London WC1N 3BG, England.

975 Tel: +44(0) 2076794297.

976 E-Mail: j.hardy@UCL.AC.UK

977

978 Prof. Dr. A. Brice

979 Research Centre of the Brain and Spine Institute

980 (CRicm UMR_S975), Pitié-Salpêtrière Hospital, 47

981 boulevard de l'Hôpital, 75013 Paris, France

982 Tel: +33157274682

983 E-Mail: alexis.brice@upmc.fr

984

985 Prof. Dr. J.C. van Swieten; Fenne Riemslagh

986 Prof. Dr. A.B. Smit: Pim van Nierop; Suzanne Miedema 
987 Dept. Neurology, ErasmusMC, 's-Gravendijkwal 230

9883015 CE Rotterdam, Netherlands.

$989 \quad$ Tel: +31107033274

990 E-Mail: j.c.vanswieten@erasmusmc.nl

991

992 Prof. Dr. P. Longone/

993 Prof. Dr. E. Buratti/

994 Prof. Dr. A. Alberici

995 Molecular Neurobiology Unit

996 Santa Lucia Foundation

997 Via del Fosso di Fiorano 64

99800179 Roma Italia

999 Tel. +39-06501703151

1000 E-Mail: p.longone@hsnatalucia.it

1001

1002 Prof. Dr. P. van Damme

1003 Laboratory for Neurobiology (Vesalius Research

1004 Center) O\&N IV Herestraat 49 - bus 912.

10053000 Leuven, Belgium

1006 Tel. +3216373181

1007 E-Mail: philip.vandamme@vib-kuleuven.be 
1009 Dr. A. Sandelin

1010 Department of Biology, Copenhagen University,

1011 Ole Maaoles vej 5,

1012 DK2200 Copenhagen, Denmark.

1013 Tel +45 35321285/ Email: albin@binf.ku.dk

1014

1015 Prof. Dr. A. Fischer

1016 Dept. of Psychiatry and Psychotherapy, University

1017 of Göttingen, German Center for Neurodegenerative

1018 Diseases (DZNE) Göttingen, Grisebach Str. 5,

101937077 Göttingen, Germany.

1020 Tel: +4955130178.

1021 E-Mail: afische2@gwdg.de

1022

1023 Prof. Dr. S. Lichtenthaler

1024 Technical University Munich (TUM) and German

1025 Center for Neurodegenerative Diseases (DZNE),

1026 Max-Lebsche Platz 30, 81377 Munich, Germany.

1027 Tel: +498970958405

1028 E-Mail: stefanlichtethaler@dzne.de 
1030 Prof. Dr. E. Mandelkow + Prof. Dr. E. Mandelkow + Marta Anglada

1031 German Center for Neurodegenerative Diseases

1032 (DZNE), Ludwig-Erhard-Allee 253175 Bonn,

1033 Germany.

1034 Tel: $+49(0) 228 / 43302-630$

1035 E-Mail: eckhard.mandelkow@dzne.de

1036

1037 Competing Interests

1038 The authors declare no competing interests.

1039 


\section{Figures}

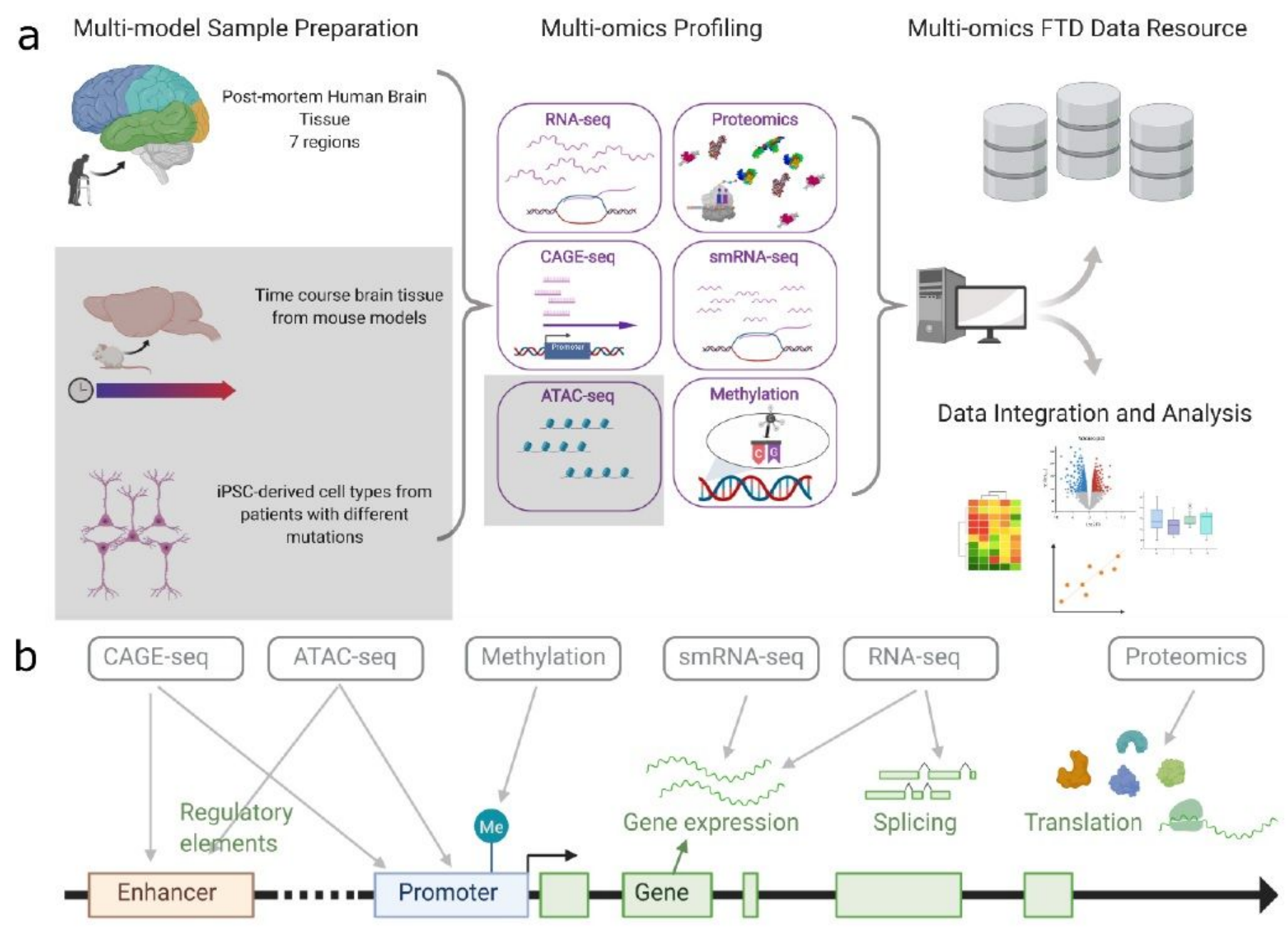

\section{Figure 1}

Graphical overview of the RiMod-FTD project. a In phase 1, Human Post mortem brain tissue samples from multiple regions of patients with mutations in GRN, MAPT and C9orf72 have been collected and used for multi-omics data generation. Grey shading indicates datasets that will be completed in future phases. The datasets have been integrated and analysed and published to be accessible as FTD resource. In subsequent phases additional datatypes will be added (i.e. ATAC-seq) and the resource will be extended with data from matching mouse models and iPSC derived celltypes. $b$ The multiomics approach allows to profile multiple regulatory features of gene expression, including enhancer- and promoter-based regulation, epigenetic regulation, alternative splicing, post transcriptional regulation (miRNAs) and regulation of translation (proteomics). 

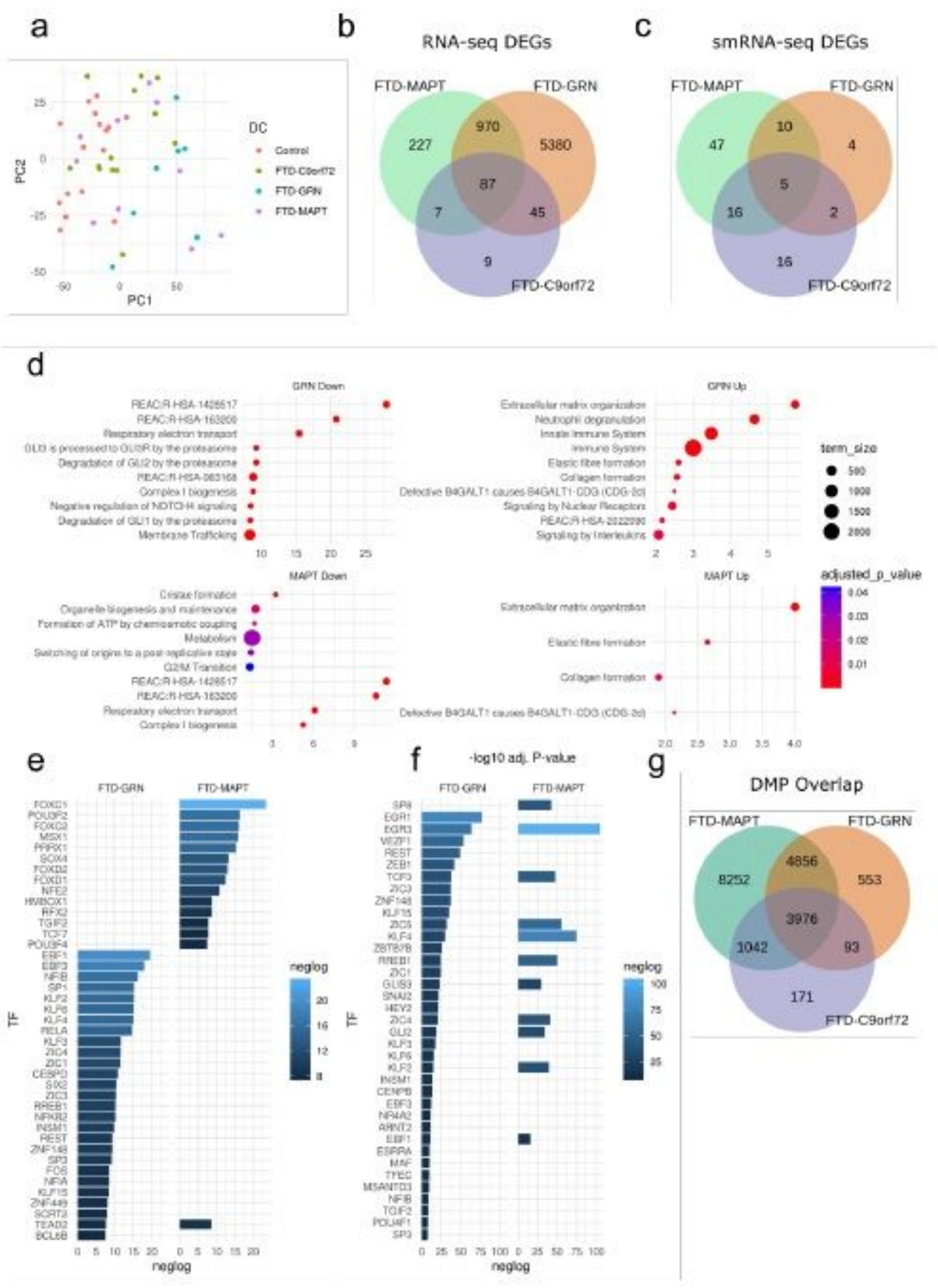

Figure 2

Gene- and Pathway-level transcriptional changes in FTD. a Principal component analysis of variance stabilized RNA-seq expression values, coloured by group. b Overlap between RNA-seq DEGs from different disease groups. c Overlap between smRNA-seq DEGs from different disease groups. $d$ Enriched Reactome pathways in RNA seq up- and down-regulated DEGs. Shown are the ten most significant pathways per group; the $\mathrm{x}$-axis signifies the negative log10 P-value. Colour corresponds to adjusted Pvalue and node size corresponds to the number of genes in a pathway. e,f Best candidates for active and 
inactive TFs in FTD-GRN and FTD-MAPT, respectively. The x-axis signifies the negative log10 P-value. $g$ Overlap of DMPs in different disease groups.

\section{a}

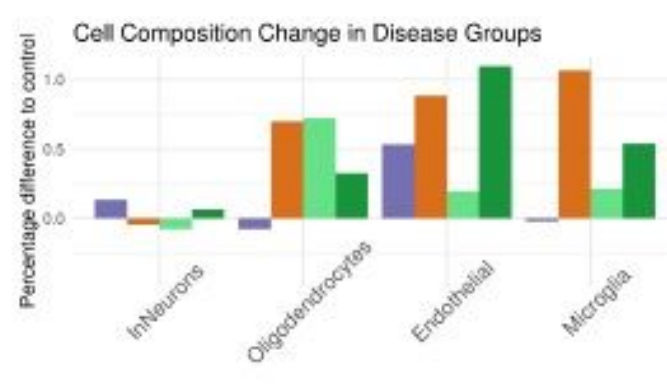

C

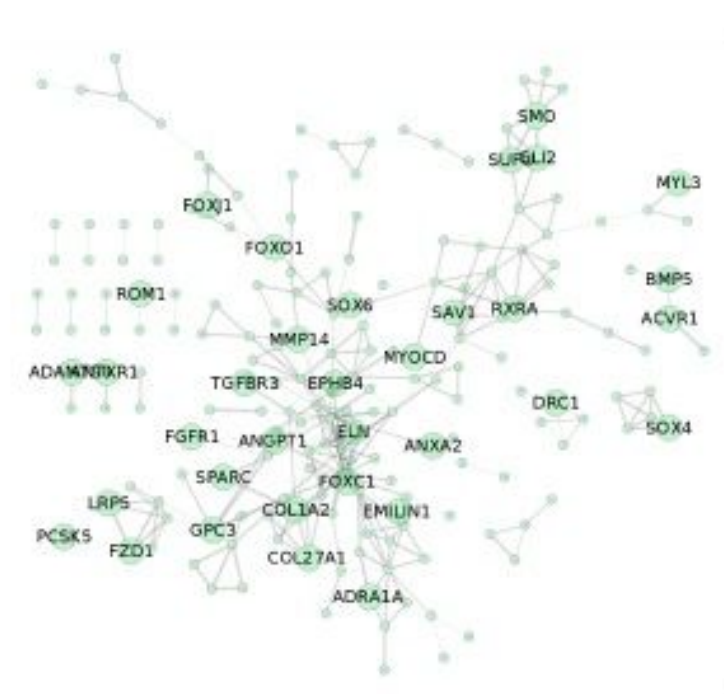

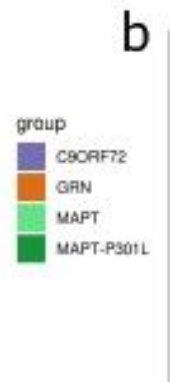

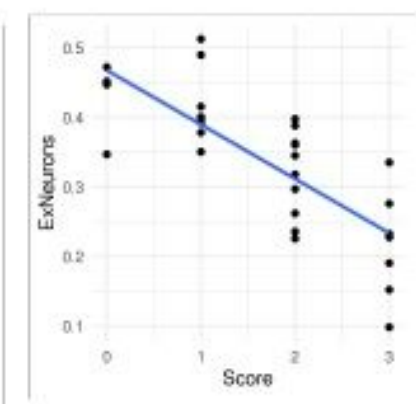

d

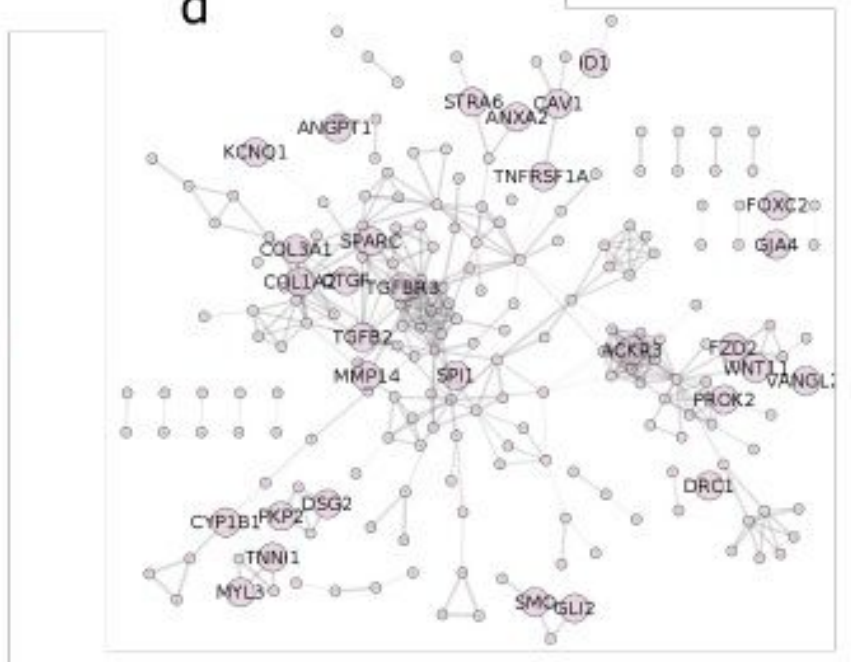

e

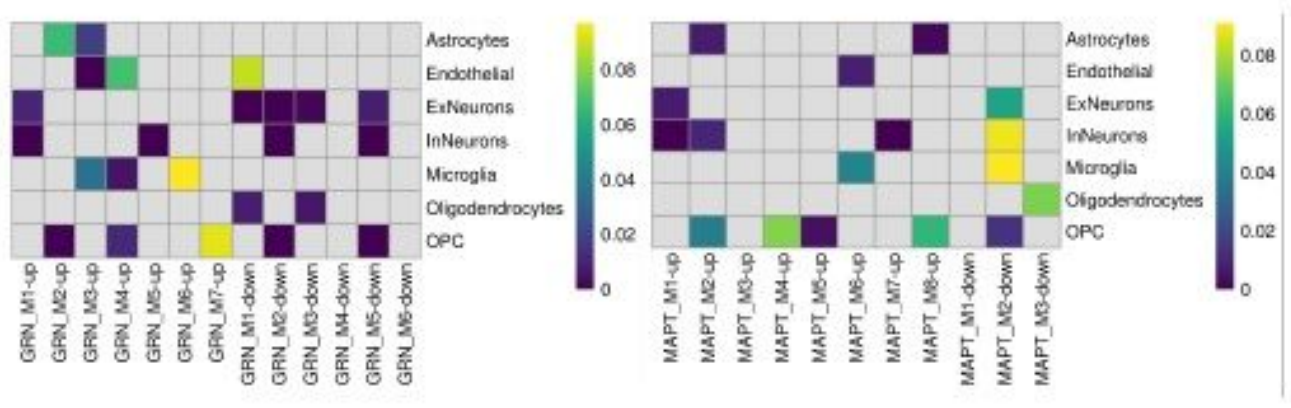

Figure 3

Cell composition changes in FTD. a Percentage change of averaged fractions per group compared to the average of the control group. Genetic subtypes are indicated with different colours. b Regression of excitatory neuron fractions (y-axis) against neuropathology scores (x-axis). c, d PPI networks of genes upregulated in FTD-MAPT and FTD-GRN (log fold-change > 1), respectively. Genes involved in the biological process "circulatory system development" are labelled. e Heatmap of EWCE analysis results for HumanBase modules of FTD-GRN and FTD-MAPT. Different modules are lined up on the $265 \mathrm{x}$-axis, different cell types on the $y$-axis. Tile colour signifies the EWCE P-value. Tiles with P-values above 0.1 are marked grey. 

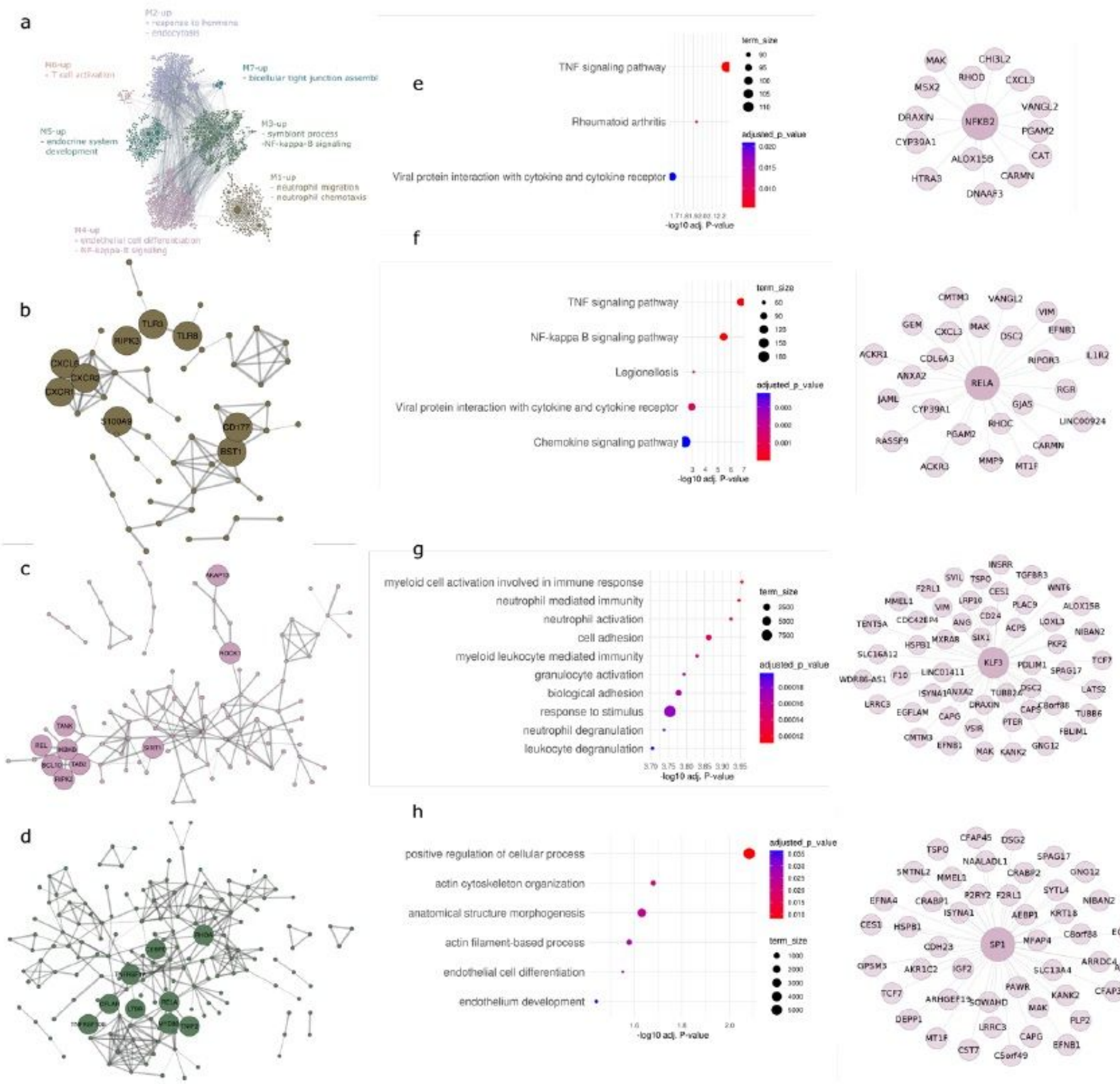

h
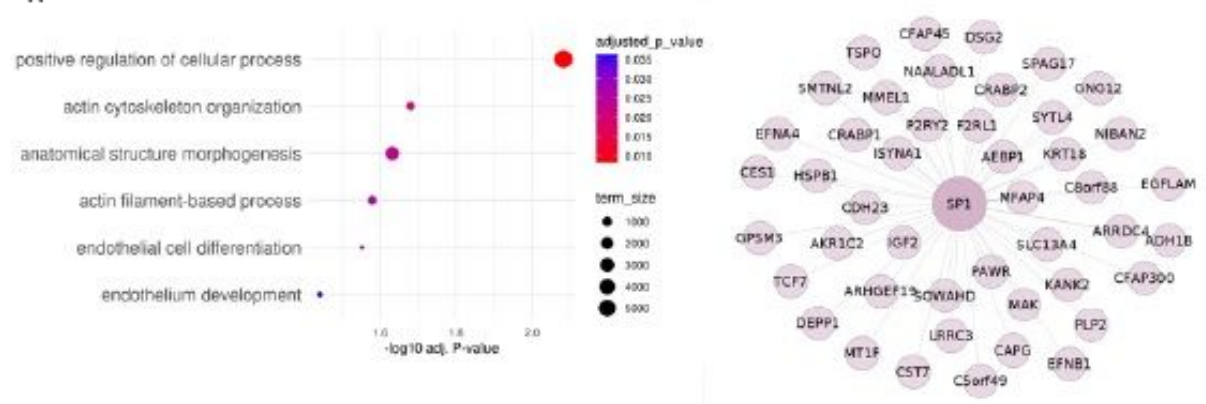

\section{Figure 4}

Neuroinflammation in FTD-GRN. a Up-regulated HumanBase modules in FTD GRN with most significant terms. b Protein-protein interaction (PPI) network (made with String-DB) of FTD-GRN M1 up-module. Genes involved in necroptosis, interleukin response and neutrophil migration are indicated c PPI network of FTD-GRN M4-up module. Genes involved in NFkB signalling are indicated. d PPI network of FTD-GRN M3-up module. Genes involved in NFkB signalling and CEPBD are indicated. e and f KEGG pathway enrichment of predicted targets of TFs NFKB2 and RELA, respectively. $g$ and $\mathrm{h}$ GO:BP pathway enrichment of predicted targets of TFs KLF3 and SP1, respectively. 


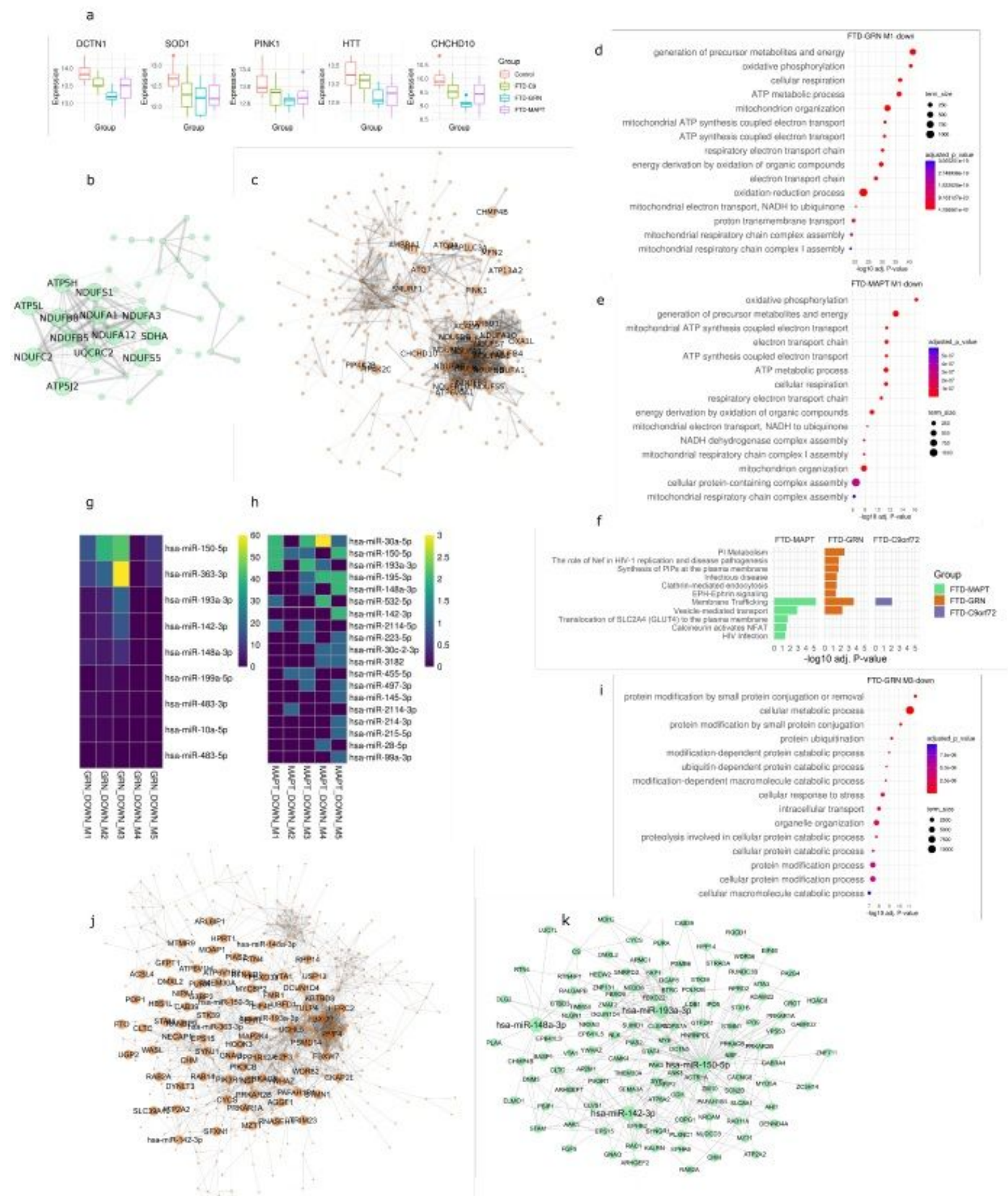

\section{Figure 5}

Impaired oxidative phosphorylation and cellular trafficking in FTD. a Expression levels (variance stabilized with DESeq2) of the genes CHCHD10, PINK1, SOD1, DCTN1 and HTT in different groups. $b$ STRING-DB PPI of FTD-MAPT M1-down module. Genes involved in oxidative phosphorylation are labelled. c PPI of FTD-GRN M1-down module. Genes involved in NADH dehydrogenase complex assembly and mitophagy are labelled, as well as CHCHD10. $d$, e Most significant results from pathway 
enrichment analysis with g:Profiler (GO biological process) for the FTD-GRN M1-down module and the FTD-MAPT M1-down module, respectively. Node colour corresponds to adjusted P-value and node size to term size. f Most significant results from enrichment analysis (Reactome) of targets of upregulated miRNAs in all disease groups. $g$, h Heatmaps of intersection-over union scores between predicted miRNA targets and down-regulated modules in FTD-GRN and FTD-MAPT, respectively. i Top enrichment results of g:Profiler (GO biological process) for the FTD-GRN M3-down module. Node colour corresponds to adjusted P-value and nodem size to term size. j PPI network (String-DB) of FTD-GRN M3down module. Predicted targets of up-regulated miRNAs are labelled. k PPI network of predicted targets of up-regulated miRNAs in FTD-MAPT.

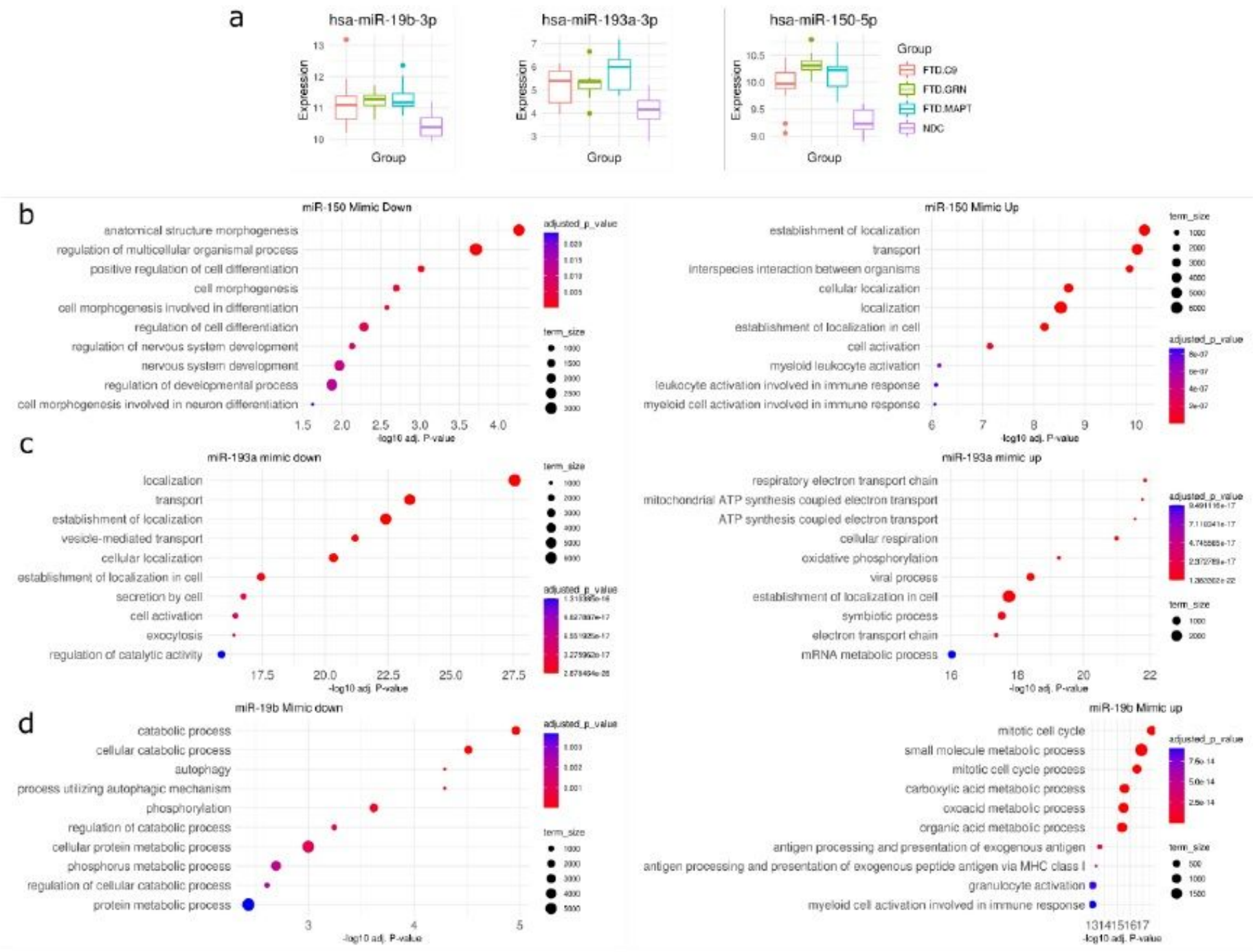

Figure 6

Effects of miRNA mimic and inhibitor experiments in iPSC-derived microglia a Boxplots of normalized expression values for the selected miRNAs. $b, c, d$ The top ten most significantly enriched biological processes of up- and down-regulated genes after transfection with mimics for miR-150-5p, miR-193a-3p and miR-19b-3p, respectively. Node size corresponds to the number of genes in the biological process term and node colour corresponds to the P-value adjusted for multiple testing. 


\section{Supplementary Files}

This is a list of supplementary files associated with this preprint. Click to download.

- rimodpapersupplements.pdf 